\begin{tabular}{|c|l|}
\hline Title & Singularities of Anti de Sitter torus Gauss maps \\
\hline Author(s) & Chen, Liang; Izumiya, Shyuichi \\
\hline Citation & $\begin{array}{l}\text { Bulletin of the Brazilian Mathematical Society, 41(1), 37-61 } \\
\text { https://doi.org/40.1007/300574_010-0002-3 }\end{array}$ \\
\hline Issue Date & 2010-03 \\
\hline Doc URL & http://hdl.handle.net/2115/42975 \\
\hline Rights & The final publication is available at www.springerlink.com \\
\hline Type & article (author version) \\
\hline File Information & BBMS41-1_37-61.pdf \\
\hline
\end{tabular}

Instructions for use 


\title{
Singularities of Anti de Sitter Torus Gauss maps
}

\author{
Liang Chen ${ }^{1,2, \dagger}$ and Shyuichi Izumiya ${ }^{2, *}$ \\ 1. School of Mathematics and Statistics, Northeast Normal University, \\ Changchun 130024, P.R.CHINA \\ chenliang@sci.math.hokudai.ac.jp, chenL234@nenu.edu.cn \\ 2. Department of Mathematics, Faculty of Science, Hokkaido University, \\ Sapporo 060-0810, JAPAN \\ Izumiya@math.sci.hokudai.ac.jp
}

\begin{abstract}
We study timelike surfaces in Anti de Sitter 3-space as an application of singularity theory. We define two mappings associated to a timelike surface which are called a Anti de Sitter nullcone Gauss image and a Anti de Sitter torus Gauss map. We also define a family of functions named the Anti de Sitter null height function on the timelike surface. We use this family of functions as a basic tool to investigate the geometric meanings of singularities of the Anti de Sitter nullcone Gauss image and the Anti de Sitter torus Gauss map.
\end{abstract}

Keywords: Anti de Sitter 3-space; timelike surface; AdS-nullcone Gauss image; AdS-torus Gauss map; Legendrian singularities.

Mathematics Subject classification: 53A35; 58C25

† Work partially supported by Science Foundation for Young Teachers of Northeast Normal University No. 20070105 and the State Scholarship Fund of CSC No. 20073021, China.

* Work partially supported by Grant-in Aid for Scientific Research No. 18654007 and No. 18340013.

Acknowledgments: The work was completed when the first author was a joint $\mathrm{PhD}$ candidate at the Hokkaido University. He would like to thank the peoples in department of mathematics for their hospitality.

Corresponding Author: chenliang@math.sci.hokudai.ac.jp, chenL234@nenu.edu.cn(It will come into use from Oct. 2008). 
Anti de Sitter Torus Gauss maps

\section{Introduction}

This paper is written as one of the research projects on differential geometry of submanifolds in Anti de Sitter 3-space from the viewpoint of singularity theory. There are several articles for the study of submanifolds in Minkowski space, which is a flat Lorentzian space, and also in de Sitter space, which is a Lorentzian space with positive curvature $[10,12,13,14,15]$. The Lorentzian space form with negative curvature is called Anti de Sitter space which is one of the vacuum solutions of the Einstein equation in the theory of relativity. Singularity theory tools, as illustrated by several papers which appeared so far $([2,4,5,6,7,9,10,11$, $16,20,21,22,23,25,28,29,30])$, have proven to be useful in the description of geometrical properties of submanifolds immersed in different ambient spaces, from both the local and global viewpoint. The natural connection between Geometry and Singularities relies on the basic fact that the contacts of a submanifold with the models (invariant under the action of a suitable transformation group) of the ambient space can be described by means of the analysis of the singularities of appropriate families of contact functions, or equivalently, of their associated Lagrangian and/or Legendrian maps $([1,24,26])$. However, there are not many results on submanifolds immersed in Anti de Sitter space, in particular from the view point of singularity theory. We remark that although Anti de Sitter space is diffeomorphic to de Sitter space, their causalities are quite different. In [8] we have studied the spacelike surfaces in Anti de Sitter 3-space as an application of Legendrian singularity theory. We construct a basic framework for the study of timelike surfaces in Anti de Sitter 3-space here. As it was to be expected, the situation presents certain peculiarities when compared with the Minkowski case and the de Sitter case. For instance, in our case it is always possible to choose two lightlike normal directions along the timelike surface in the frame of its normal bundle. This is similar to the de Sitter case, but the normalized image is located in the Lorentzian torus $T_{1}^{2}$. For the de Sitter case, the normalized image of the lightlike normal is located in the spacelike sphere $S_{+}^{2}$. Moreover, there are no closed timelike surfaces in de Sitter space but there are such surfaces in Anti de Sitter space.

In $\S 2$ we prepare the basic notions on timelike surfaces in Anti de Sitter 3-space. We define the Anti de Sitter nullcone Gauss image (briefly, AdS-nullcone Gauss image) and Anti de Sitter torus Gauss map (briefly, AdS-torus Gauss map). We will find the AdS-nullcone Gauss image is more computable than the AdS-torus Gauss map. We also define the Anti de Sitter null GaussKronecker curvature and Anti de Sitter torus Gauss-Kronecker curvature. We investigate their relations. We can prove that Anti de Sitter torus Gauss-Kronecker curvature is not a Lorentz invariant but it is an $S O(2) \times S O(2)$-invariant. Moreover, these two Gauss-Kronecker curvature functions have the same zero sets. In $\S 3$ we introduce the notion of height functions on timelike surfaces, named AdS-null height function, which is useful to show that the AdS-nullcone Gauss image has a singular point if and only if the Anti de Sitter null Gauss-Kronecker curvature vanished at such point. we also apply the Legendrian singularity theory to interpret the AdSnullcone Gauss image as a Legendrian map. In $\S 4$ we define a surface, named Anti de Sitter torus cylindrical pedal, as a tool to study the relationship between the AdS-nullcone Gauss image and the AdS-torus Gauss map. We also study the contact of timelike surfaces with some model surfaces (i.e., AdS-horospheres) in $\S 5$. In $\S 6$ we give a generic classification of singularities of AdS-nullcone Gauss image and AdS-torus Gauss map. In the last part, $\S 7$, we introduce the notion of the AdS-null Monge form of a timelike surface in Anti de Sitter 3-space and as an application of this notion we give two examples. 
We shall assume throughout the whole paper that all maps and manifolds are $C^{\infty}$ unless the contrary is explicitly stated.

\section{The local differential geometry of timelike surfaces}

In this section we introduce the local differential geometry of timelike surfaces in Anti de Sitter 3 -space. For details of Lorentzian geometry, see [27].

Let $\mathbb{R}^{4}=\left\{\left(x_{1}, \cdots, x_{4}\right) \mid x_{i} \in \mathbb{R}(i=1, \cdots, 4)\right\}$ be a 4-dimensional vector space. For any vectors $\boldsymbol{x}=\left(x_{1}, \cdots, x_{4}\right)$ and $\boldsymbol{y}=\left(y_{1}, \cdots, y_{4}\right)$ in $\mathbb{R}^{4}$, the pseudo scalar product of $\boldsymbol{x}$ and $\boldsymbol{y}$ is defined to be $\langle\boldsymbol{x}, \boldsymbol{y}\rangle=-x_{1} y_{1}-x_{2} y_{2}+x_{3} y_{3}+x_{4} y_{4}$. We call $\left(\mathbb{R}^{4},\langle\rangle,\right)$ a semi-Euclidean 4 -space with index 2 and write $\mathbb{R}_{2}^{4}$ instead of $\left(\mathbb{R}^{4},\langle\rangle,\right)$.

We say that a non-zero vector $\boldsymbol{x}$ in $\mathbb{R}_{2}^{4}$ is spacelike, null or timelike if $\langle\boldsymbol{x}, \boldsymbol{x}\rangle>0,\langle\boldsymbol{x}, \boldsymbol{x}\rangle=0$ or $\langle\boldsymbol{x}, \boldsymbol{x}\rangle<0$ respectively. The norm of the vector $\boldsymbol{x} \in \mathbb{R}_{2}^{4}$ is defined by $\|\boldsymbol{x}\|=\sqrt{|\langle\boldsymbol{x}, \boldsymbol{x}\rangle|}$. We denote the signature of a vector $\boldsymbol{x}$ by

$$
\operatorname{sign}(\boldsymbol{x})= \begin{cases}1 & \boldsymbol{x} \text { is spacelike } \\ 0 & \boldsymbol{x} \text { is null } \\ -1 & \boldsymbol{x} \text { is timelike }\end{cases}
$$

For a vector $\boldsymbol{n} \in \mathbb{R}_{2}^{4}$ and a real number $c$, we define the hyperplane with pseudo-normal $\boldsymbol{n}$ by

$$
H P(\boldsymbol{n}, c)=\left\{\boldsymbol{x} \in \mathbb{R}_{2}^{4} \mid\langle\boldsymbol{x}, \boldsymbol{n}\rangle=c\right\} .
$$

We call $H P(\boldsymbol{n}, c)$ a Lorentz hyperplane, a semi-Euclidean hyperplane with index 2 or a null hyperplane if $\boldsymbol{n}$ is timelike, spacelike or null respectively.

We now define Anti de Sitter 3-space (briefly, AdS 3-space) by

$$
H_{1}^{3}=\left\{\boldsymbol{x} \in \mathbb{R}_{2}^{4} \mid\langle\boldsymbol{x}, \boldsymbol{x}\rangle=-1\right\},
$$

a unit pseudo 3-sphere with index 2 by

$$
S_{2}^{3}=\left\{\boldsymbol{x} \in \mathbb{R}_{2}^{4} \mid\langle\boldsymbol{x}, \boldsymbol{x}\rangle=1\right\},
$$

and a closed nullcone with vertex $\boldsymbol{a}$ by

$$
\Lambda_{a}=\left\{\boldsymbol{x} \in \mathbb{R}_{2}^{4} \mid\langle\boldsymbol{x}-\boldsymbol{a}, \boldsymbol{x}-\boldsymbol{a}\rangle=0\right\} .
$$

In particular we call $\Lambda_{0}$ the nullcone at the origin. We also define the Lorentz torus by

$$
T_{1}^{2}=\left\{\boldsymbol{x}=\left(x_{1}, x_{2}, x_{3}, x_{4}\right) \in \Lambda_{0} \mid x_{1}^{2}+x_{2}^{2}=x_{3}^{2}+x_{4}^{2}=1\right\} .
$$

If a non-zero vector $\boldsymbol{x}=\left(x_{1}, x_{2}, x_{3}, x_{4}\right) \in \Lambda_{0}$, we have

$$
\tilde{\boldsymbol{x}}= \pm \frac{1}{\sqrt{x_{1}^{2}+x_{2}^{2}}}\left(x_{1}, x_{2}, x_{3}, x_{4}\right)= \pm \frac{1}{\sqrt{x_{1}^{2}+x_{2}^{2}}} \boldsymbol{x} \in T_{1}^{2}
$$

For any $\boldsymbol{X}_{1}, \boldsymbol{X}_{2}, \boldsymbol{X}_{3} \in \mathbb{R}_{2}^{4}$, we define a vector $\boldsymbol{X}_{1} \wedge \boldsymbol{X}_{2} \wedge \boldsymbol{X}_{3}$ by

$$
\boldsymbol{X}_{1} \wedge \boldsymbol{X}_{2} \wedge \boldsymbol{X}_{3}=\left|\begin{array}{cccc}
-\boldsymbol{e}_{1} & -\boldsymbol{e}_{2} & \boldsymbol{e}_{3} & \boldsymbol{e}_{4} \\
x_{1}^{1} & x_{1}^{2} & x_{1}^{3} & x_{1}^{4} \\
x_{2}^{1} & x_{2}^{2} & x_{2}^{3} & x_{2}^{4} \\
x_{3}^{1} & x_{3}^{2} & x_{3}^{3} & x_{3}^{4}
\end{array}\right|
$$


where $\left\{\boldsymbol{e}_{1}, \boldsymbol{e}_{2}, \boldsymbol{e}_{3}, \boldsymbol{e}_{4}\right\}$ is the canonical basis of $\mathbb{R}_{2}^{4}$ and $\boldsymbol{X}_{i}=\left(x_{i}^{1}, x_{i}^{2}, x_{i}^{3}, x_{i}^{4}\right)$. We can easily check that $\left\langle\boldsymbol{X}, \boldsymbol{X}_{1} \wedge \boldsymbol{X}_{2} \wedge \boldsymbol{X}_{3}\right\rangle=\operatorname{det}\left(\boldsymbol{X}, \boldsymbol{X}_{1}, \boldsymbol{X}_{2}, \boldsymbol{X}_{3}\right)$, so that $\boldsymbol{X}_{1} \wedge \boldsymbol{X}_{2} \wedge \boldsymbol{X}_{3}$ is pseudo-orthogonal to any $\boldsymbol{X}_{i}$ (for $\left.i=1,2,3\right)$.

We now study the extrinsic differential geometry of timelike surfaces in Anti de Sitter 3space. Let $\boldsymbol{X}: U \longrightarrow H_{1}^{3}$ be a regular surface (i.e., an embedding), where $U \subset \mathbb{R}^{2}$ is an open subset. We denote $M=\boldsymbol{X}(U)$ and identify $M$ with $U$ through the embedding $\boldsymbol{X}$. The embedding $\boldsymbol{X}$ is said to be timelike if the induced metric $\mathbf{I}$ of $M$ is Lorentzian. Throughout the remainder in this paper we assume that $M$ is a timelike surface in $H_{1}^{3}$. We define a vector $\mathbf{N}(u)$ by

$$
\boldsymbol{N}(u)=\frac{\boldsymbol{X}(u) \wedge \boldsymbol{X}_{u_{1}}(u) \wedge \boldsymbol{X}_{u_{2}}(u)}{\left\|\boldsymbol{X}(u) \wedge \boldsymbol{X}_{u_{1}}(u) \wedge \boldsymbol{X}_{u_{2}}(u)\right\|}
$$

By definition, we have

$$
\langle\boldsymbol{N}(u), \boldsymbol{X}(u)\rangle \equiv\left\langle\boldsymbol{N}(u), \boldsymbol{X}_{u_{i}}(u)\right\rangle \equiv 0 \text { and }\left\langle\boldsymbol{X}(u), \boldsymbol{X}_{u_{i}}(u)\right\rangle \equiv 0(\text { for } i=1,2) .
$$

This means that $\boldsymbol{X}(u), \boldsymbol{N}(u) \in N_{p} M$, where $u=\left(u_{1}, u_{2}\right) \in U$ and $p=\boldsymbol{X}(u) \in M$. Since the embedding is timelike and $\boldsymbol{X}(u) \in H_{1}^{3}, \boldsymbol{N}$ is spacelike. Therefore $\langle\boldsymbol{N}(u), \boldsymbol{N}(u)\rangle \equiv 1$. It follows that $\boldsymbol{X}(u) \pm \boldsymbol{N}(u) \in \Lambda_{0} \cap N_{p} M$ and $\boldsymbol{X}(u) \pm \boldsymbol{N}(u) \in T_{1}^{2} \cap N_{p} M$. Thus we can define a map $\mathbb{G}_{n}^{ \pm}: U \longrightarrow \Lambda_{0}$ by $\mathbb{G}_{n}^{ \pm}(u)=\boldsymbol{X}(u) \pm \boldsymbol{N}(u)$. This map is analogous to the hyperbolic Gauss indicatrix of hypersurfaces in $H_{+}^{n}(-1)$ which was defined in [11]. Here, we call it the Anti de Sitter nullcone Gauss image (briefly, AdS-nullcone Gauss image) of $\boldsymbol{X}$ (or $M)$. We also define a map $\widetilde{\mathbb{G}_{n}^{ \pm}}: U \longrightarrow T_{1}^{2}$ by $\widetilde{\mathbb{G}_{n}^{ \pm}}(u)=\boldsymbol{X}\left(\widetilde{u) \pm \boldsymbol{N}}(u)=\frac{1}{\xi(u)} \mathbb{G}_{n}^{ \pm}(u)\right.$, where $\xi(u)= \pm \sqrt{\left(x_{1}(u) \pm n_{1}(u)\right)^{2}+\left(x_{2}(u) \pm n_{2}(u)\right)^{2}}$. We call it the Anti de Sitter torus Gauss map (or, AdS-torus Gauss map) of $\boldsymbol{X}$.

We remak that the map $\mathbb{G}_{n}^{ \pm}(u)$ was used by S. Lee [17] to study the timelike sufaces of constant mean curvature \pm 1 in Anti de Sitter 3 -space. He called $\mathbb{G}_{n}^{ \pm}(u)$ the hyperbolic Gauss map. By a direct calculation we know that $\mathbb{G}_{n}^{ \pm}$is constant if and only if $\widetilde{\mathbb{G}_{n}^{ \pm}}$is constant.

It is easy to show that $\boldsymbol{N}_{u_{i}}(i=1,2)$ are tangent vectors of $M$. Therefore we have a linear transformation $S_{p}^{ \pm}=-d \mathbb{G}_{n}^{ \pm}(u)=-(d \boldsymbol{X}(u) \pm d \boldsymbol{N}(u)): T_{p} M \longrightarrow T_{p} M$ which is called the Anti de Sitter null shape operator (briefly, AdS-null shape operator) of $M=\boldsymbol{X}(U)$ at $p=\boldsymbol{X}(u)$. Under the identification of $U$ and $M$, the derivation $d \boldsymbol{X}(u)$ can be identified with the identity mapping $\operatorname{id}_{T_{p} M}$, this means that $S_{p}^{ \pm}=-d \mathbb{G}_{n}^{ \pm}(u)=-\left(\operatorname{id}_{T_{p} M} \pm d \boldsymbol{N}(u)\right)$. We have another linear mapping

$$
d \widetilde{\mathbb{G}_{n}^{ \pm}}(u): T_{p} M \longrightarrow T_{p} \mathbb{R}_{2}^{4}=T_{p} M \oplus N_{p} M
$$

If we consider the orthogonal projection $\pi^{T}: T_{p} M \oplus N_{p} M \longrightarrow T_{p} M$, then we have

$$
\widetilde{S_{p}^{ \pm}}=-\left(d \widetilde{\mathbb{G}_{n}^{ \pm}}(u)\right)^{T}=-\pi^{T} \circ d \widetilde{\mathbb{G}_{n}^{ \pm}}(u): T_{p} M \longrightarrow T_{p} M
$$

and call it the Anti de Sitter torus shape operator (briefly, AdS-torus shape operator) of $M=$ $\boldsymbol{X}(U)$ at $p=\boldsymbol{X}(u)$. We remark that $S_{p}^{ \pm}$(resp., $\widetilde{S_{p}^{ \pm}}$) does not always have real eigenvalues. If the eigenvalues are real numbers, we denote it by $k_{i}^{ \pm}$(resp., $\left.\widetilde{k_{i}^{ \pm}}\right)($for $i=1,2)$.

We define $K_{A d S n}^{ \pm}(u)=\operatorname{det} S_{p}^{ \pm}=k_{1}^{ \pm} \cdot k_{2}^{ \pm}$and $\widetilde{K_{A d S t}^{ \pm}}(u)=\operatorname{det} \widetilde{S_{p}^{ \pm}}=\widetilde{k_{1}^{ \pm}} \cdot \widetilde{k_{2}^{ \pm}}$. We respectively call $K_{A d S n}^{ \pm}(u)$ the Anti de Sitter null Gauss-Kronecker curvature (briefly, AdS-null G-K curvature) and $\widehat{K_{A d S t}^{ \pm}}(u)$ the Anti de Sitter torus Gauss-Kronecker curvature (briefly, AdS-torus $G$-K curvature) of $M=\boldsymbol{X}(U)$ at $p=\boldsymbol{X}(u)$. We say that a point $p=\boldsymbol{X}(u)$ is a (positive or negative) Anti de Sitter horospherical parabolic point (briefly, AdSh ${ }^{ \pm}$-parabolic point) (resp. positive or negative Anti de Sitter torus parabolic point, briefly, AdSt $t^{ \pm}$-parabolic point) of $M=\boldsymbol{X}(U)$ 
if $K_{A d S n}^{ \pm}(u)=0\left(\right.$ resp. $\left.\widetilde{K_{A d S t}^{ \pm}}(u)=0\right)$. By a straightforward calculation we have the relation $S_{p}^{ \pm}=\xi(u) \widetilde{S_{p}^{ \pm}}$, so that we have $k_{i}^{ \pm}(p)=\xi(u) \widetilde{k_{i}^{ \pm}}(p)$ and $K_{A d S n}^{ \pm}(u)=\xi^{2}(u) \widetilde{K_{A d S t}^{ \pm}}(u)$. Then we have the following relations:

$$
\left\{\begin{array}{l}
k_{i}^{ \pm}(p)=0 \Longleftrightarrow \widetilde{k_{i}^{ \pm}}(u)=0 \\
K_{A d S n}^{ \pm}(u)=0 \Longleftrightarrow \widetilde{K_{A d S t}^{ \pm}}(u)=0 .
\end{array}\right.
$$

We say that a point $u \in U$ or $p=\boldsymbol{X}(u)$ is an umbilic point if $S_{p}^{ \pm}=k^{ \pm}(p) i d_{T_{p} M}$. We also say that $M=\boldsymbol{X}(U)$ is totally umbilic if all points on $M$ are umbilic.

We now consider the geometric meaning of the AdS-nullcone Gauss image of a timelike surface. First, we consider a surface given by the intersection of $H_{1}^{3}$ with hyperplane $H P(\boldsymbol{n}, c)$. We denote it by $A H(\boldsymbol{n}, c)=H_{1}^{3} \cap H P(\boldsymbol{n}, c)$ and call it a Anti de Sitter pseudohyperboloid with index 1 (briefly, AdS-pseudohyperboloid), a Anti de Sitter pseudosphere with index 1 (briefly, AdS-pseudosphere) or a Anti de Sitter horosphere (briefly, AdS-horosphere) if $\boldsymbol{n}$ is spacelike, timelike and $\|\boldsymbol{n}\|<|c|$ or null respectively. Especially, we call $A H(\boldsymbol{n}, 0)$ the Anti de Sitter small pseudohyperboloid with index 1 (briefly, AdS-small pseudohyperboloid) if $\boldsymbol{n}$ is spacelike and $c=0$. Then we have the following proposition.

Proposition 2.1 Let $\boldsymbol{X}: U \longrightarrow H_{1}^{3}$ be a timelike surface in Anti de Sitter 3-space. If the AdS-nullcone Gauss image $\mathbb{G}_{n}^{ \pm}$is constant, then the timelike surface $\boldsymbol{X}(U)=M$ is a part of a AdS-horosphere.

Proof. We consider the set $V=\left\{\boldsymbol{y} \in \mathbb{R}_{2}^{4} \mid\langle\boldsymbol{y}, \boldsymbol{X} \pm \boldsymbol{N}\rangle=-1\right\}$. Since $\mathbb{G}_{n}^{ \pm}=\boldsymbol{X} \pm \boldsymbol{N}$ is constant, the set $V=H P\left(\mathbb{G}_{n}^{ \pm},-1\right)$ is a null hyperplane. We also have $\left\langle\boldsymbol{X}, \mathbb{G}_{n}^{ \pm}\right\rangle \equiv-1$, so $\boldsymbol{X}(U)=M \subset V \cap H_{1}^{3}$.

We also have the following classification theorem on umbilic points.

Proposition 2.2 Suppose that $M=\boldsymbol{X}(U)$ is totally umbilic. Then $k^{ \pm}(p)$ is constant $k^{ \pm}$. Under this condition, we have the following classification.

(1) Suppose $k^{ \pm} \neq 0$.

(a) If $0<\left|k^{ \pm}+1\right|<1$, then $M$ is a part of a AdS-pseudohyperboloid;

(b) If $\left|k^{ \pm}+1\right|>1$, then $M$ is a part of a AdS-pseudosphere;

(c) If $k^{ \pm}=-1$, then $M$ is a part of a AdS-small pseudohyperboloid.

(2) Suppose $k^{ \pm}=0$ then $M$ is a part of a AdS-horosphere.

The proof is almost the same as that of Proposition 2.3 in [11], so that we omit it. We also call a point $p \in M$ the Anti de Sitter horospherical point (briefly, AdS-horospherical point) if $k_{i}^{ \pm}(p)=0(i=1,2)$.

We now introduce the pseudo-Riemannian metric $d s^{2}=\sum_{i, j=1}^{2} g_{i j} d u_{i} d u_{j}$ on $M=\boldsymbol{X}(U)$, where $g_{i j}(u)=\left\langle\boldsymbol{X}_{u_{i}}(u), \boldsymbol{X}_{u_{j}}(u)\right\rangle$ for any $u \in U$. We also define the Anti de Sitter null second fundamental invariant by $h_{i j}^{ \pm}(u)=\left\langle-\left(\mathbb{G}_{n}^{ \pm}\right)_{u_{i}}(u), \boldsymbol{X}_{u_{j}}(u)\right\rangle$, Anti de Sitter torus second fundamental invariant by $\widetilde{h_{i j}^{ \pm}}(u)=\left\langle-\left(\widetilde{\mathbb{G}_{n}^{ \pm}}\right)_{u_{i}}(u), \boldsymbol{X}_{u_{j}}(u)\right\rangle=\frac{1}{\xi(u)} h_{i j}^{ \pm}(u)$ for any $u \in U$. We can also show the following Weingarten formulas by exactly the same arguments as those of $[8,11,15]$.

Proposition 2.3 With the above notations the following hold

(1) The Anti de Sitter null Weingarten formula: 


$$
\left(\mathbb{G}_{n}^{ \pm}\right)_{u_{i}}=-\sum_{j=1}^{2}\left(h^{ \pm}\right)_{i}^{j} \boldsymbol{X}_{u_{j}}
$$

where $\left(\left(h^{ \pm}\right)_{i}^{j}\right)=\left(h_{i k}^{ \pm}\right)\left(g^{k j}\right)$ and $\left(g^{k j}\right)=\left(g_{k j}\right)^{-1}$.

(2) The Anti de Sitter torus Weingarten formula:

$$
\left(\left(\widetilde{\mathbb{G}_{n}^{ \pm}}\right)_{u_{i}}\right)^{T}=\pi^{T} \circ\left(\widetilde{\mathbb{G}_{n}^{ \pm}}\right)_{u_{i}}=-\sum_{j=1}^{2}\left(\widetilde{h^{ \pm}}\right)_{i}^{j} \boldsymbol{X}_{u_{j}}=-\frac{1}{\xi(u)} \sum_{j=1}^{2}\left(h^{ \pm}\right)_{i}^{j} \boldsymbol{X}_{u_{j}},
$$

where $\left.\left(\widetilde{\left(h^{ \pm}\right.}\right)_{i}^{j}\right)=\left(\widetilde{h_{i k}^{ \pm}}\right)\left(g^{k j}\right)$ and $\left(g^{k j}\right)=\left(g_{k j}\right)^{-1}$.

As a corollary of the above proposition, we have the following expression of the AdS-null G-K curvature and AdS-torus G-K curvature .

Corollary 2.4 With the same notations as in the above Proposition, we have:

$$
K_{A d S n}^{ \pm}=\frac{\operatorname{det}\left(h_{i j}^{ \pm}\right)}{\operatorname{det}\left(g_{i j}\right)}=\xi^{2} \frac{\operatorname{det}\left(\widetilde{h_{i j}^{ \pm}}\right)}{\operatorname{det}\left(g_{i j}\right)}=\xi^{2} \widetilde{K_{A d S t}^{ \pm}} .
$$

\section{Height functions on timelike surfaces}

In this section we define two families of functions on a timelike surface in Anti de Sitter 3-space which are useful for the study of singularities of AdS-nullcone Gauss image and AdS-torus Gauss map.

Let $\boldsymbol{X}: U \longrightarrow H_{1}^{3}$ be a timelike surface. We define a family of functions $H: U \times \Lambda_{0} \longrightarrow \mathbb{R}$ by $H(u, \boldsymbol{v})=\langle\boldsymbol{X}(u), \boldsymbol{v}\rangle+1$. We call $H$ an Anti de Sitter null height function (or, AdS-null height function) on $M=\boldsymbol{X}(U)$. We denote the Hessian matrix of the AdS-null height function $h_{v_{0}}(u)=H\left(u, \boldsymbol{v}_{0}\right)$ at $u_{0}$ by $\operatorname{Hess}\left(h_{v_{0}}\right)\left(u_{0}\right)$. Then we have the following proposition.

Proposition 3.1 Let $M=\boldsymbol{X}(U)$ be a timelike surface in $H_{1}^{3}$ and $H: U \times \Lambda_{0} \longrightarrow \mathbb{R}$ be an AdS-null height function. Then we have the following:

(1) $H\left(u_{0}, \boldsymbol{v}\right)=\frac{\partial H}{\partial u_{i}}\left(u_{0}, \boldsymbol{v}\right)=0$ (for $i=1,2$ ) if and only if $\boldsymbol{v}=\boldsymbol{X}\left(u_{0}\right) \pm \boldsymbol{N}\left(u_{0}\right)=\mathbb{G}_{n}^{ \pm}\left(u_{0}\right)$;

(2) Let $\boldsymbol{v}_{0}^{ \pm}=\boldsymbol{X}\left(u_{0}\right) \pm \boldsymbol{N}\left(u_{0}\right)$, then $p=\boldsymbol{X}\left(u_{0}\right)$ is an AdSh $h^{ \pm}$parabolic point if and only if $\operatorname{detHess}\left(h_{v_{0}^{ \pm}}\right)\left(u_{0}\right)=0$;

(3) Let $\boldsymbol{v}_{0}^{ \pm}=\boldsymbol{X}\left(u_{0}\right) \pm \boldsymbol{N}\left(u_{0}\right)$, then $p=\boldsymbol{X}\left(u_{0}\right)$ is an AdS-horospherical point if and only if $\operatorname{rankHess}\left(h_{v_{0}^{ \pm}}\right)\left(u_{0}\right)=0$.

Proof. (1) Since $\left\{\boldsymbol{X}, \boldsymbol{N}, \boldsymbol{X}_{u_{1}}, \boldsymbol{X}_{u_{2}}\right\}$ is a basis of the vector space $T_{p} \mathbb{R}_{2}^{4}$ where $p=\boldsymbol{X}(u)$, there exist real numbers $\lambda, \eta, \alpha_{1}, \alpha_{2}$ such that $\boldsymbol{v}=\lambda \boldsymbol{X}+\eta \boldsymbol{N}+\alpha_{1} \boldsymbol{X}_{u_{1}}+\alpha_{2} \boldsymbol{X}_{u_{2}}$. Therefore $H(u, \boldsymbol{v})=0$ if and only if $\lambda=-\langle\boldsymbol{X}(u), \boldsymbol{v}\rangle=1$. Since $0=\frac{\partial H}{\partial u_{i}}(u, \boldsymbol{v})=\left\langle\boldsymbol{X}_{u_{i}}, \boldsymbol{v}\right\rangle=\sum_{j=1}^{2} g_{i j} \alpha_{i}$ and $\left(g_{i j}\right)$ is non-degenerate, we have $\alpha_{i}=0$ (for $i=1,2$ ). Therefore we have $\boldsymbol{v}=\boldsymbol{X}+\eta \boldsymbol{N}$. From the fact that $\langle\boldsymbol{v}, \boldsymbol{v}\rangle=0$, we have $\eta= \pm 1$.

(2) By definition, we have

$$
\operatorname{Hess}\left(h_{v_{0}^{ \pm}}\right)\left(u_{0}\right)=\left(\left\langle\boldsymbol{X}_{u_{i} u_{j}}\left(u_{0}\right), \mathbb{G}_{n}^{ \pm}\left(u_{0}\right)\right\rangle\right)=\left(-\left\langle\boldsymbol{X}_{u_{i}}\left(u_{0}\right), \mathbb{G}_{n}^{ \pm} u_{j}\left(u_{0}\right)\right\rangle\right) .
$$

From the AdS-null Weingarten formula, we have

$$
-\left\langle\boldsymbol{X}_{u_{i}},\left(\mathbb{G}_{n}^{ \pm}\right)_{u_{j}}\right\rangle=\sum_{\alpha=1}^{2}\left(h^{ \pm}\right)_{i}^{\alpha}\left\langle\boldsymbol{X}_{u_{\alpha}}, \boldsymbol{X}_{u_{j}}\right\rangle=\sum_{\alpha=1}^{2}\left(h^{ \pm}\right)_{i}^{\alpha} g_{\alpha j}=h_{i j}^{ \pm} .
$$


Therefore we have

Then assertion (2) is satisfied.

$$
K_{A d S n}^{ \pm}\left(u_{0}\right)=\frac{\operatorname{det}\left(h_{i, j}^{ \pm}\left(u_{0}\right)\right)}{\operatorname{det}\left(g_{i j}\left(u_{0}\right)\right)}=\frac{\operatorname{det} \operatorname{Hess}\left(h_{v_{0}^{ \pm}}\right)\left(u_{0}\right)}{\operatorname{det}\left(g_{i j}\left(u_{0}\right)\right)}
$$

(3) By the AdS-null Weingarten formula, $p$ is an umbilic point if and only if there exists an orthogonal matrix $A$ such that $A^{t}\left(\left(h^{ \pm}\right)_{i}^{l}\right) A=k^{ \pm} I$. Therefore, we have $\left(\left(h^{ \pm}\right)_{i}^{l}\right)=A k^{ \pm} I A^{t}=$ $k^{ \pm} I$. Then we have

$$
\operatorname{Hess}\left(h_{v_{0}^{ \pm}}\right)\left(u_{0}\right)=\left(h_{i j}^{ \pm}\left(u_{0}\right)\right)=\left(\left(h^{ \pm}\right)_{i}^{l}\left(u_{0}\right)\right)\left(g_{l j}\left(u_{0}\right)\right)=k^{ \pm}\left(g_{i j}\left(u_{0}\right)\right) .
$$

Thus, $p=\boldsymbol{X}\left(u_{0}\right)$ is a AdS-horospherical point if and only if $\operatorname{rankHess}\left(h_{v_{0}^{ \pm}}\right)\left(u_{0}\right)=0$.

As an application of the above proposition, we have the following direct corollary.

Corollary 3.2 Let $H: U \times \Lambda_{0} \longrightarrow \mathbb{R}$, with $H(u, v)=h_{v}(u)$ be an AdS-null height function on a timelike surface $M=\boldsymbol{X}(U)$ and $\mathbb{G}_{n}^{ \pm}$be the AdS-nullcone Gauss image, $p=\boldsymbol{X}(u)$. Suppose $\boldsymbol{v}^{ \pm}=G_{n}^{ \pm}(u)$, then the following conditions are equivalent:

(1) $p \in M$ is a degenerate singular point of AdS-null height function $h_{v^{ \pm}}$

(2) $p \in M$ is a singular point of AdS-nullcone Gauss image $\mathbb{G}_{n}^{ \pm}$;

(3) $K_{A d S n}^{ \pm}(u)=0$.

We can also define another family of functions $\widetilde{H}: U \times T_{1}^{2} \longrightarrow \mathbb{R}$ by $\widetilde{H}(u, \boldsymbol{v})=\langle\boldsymbol{X}(u), \boldsymbol{v}\rangle$. We call $\widetilde{H}$ an Anti de Sitter torus height function (briefly, AdS-torus height function) on $\boldsymbol{X}$. We denote the Hessian matrix of the AdS-torus height function $\widetilde{h_{v_{0}}}(u)=\widetilde{H}\left(u, \boldsymbol{v}_{0}\right)$ at $u_{0}$ by $\operatorname{Hess}\left(\widetilde{h_{v_{0}}}\right)\left(u_{0}\right)$. We remark that this family satisfies the same properties as those stated in Proposition 3.1 and Corollary 3.2.

On the other hand, we can naturally interpret the AdS-nullcone Gauss image $\mathbb{G}_{n}^{ \pm}$of $M$ as a Legendrian map from the viewpoint of generating family. For notations and some basic results on generating family, please refer to Arnold and Zakalyukin [1, 32]. Then, we have the following principle property with respect to the AdS-null height function $H$.

Proposition 3.3 The AdS-null height function $H: U \times \Lambda_{0} \longrightarrow \mathbb{R}$ is a Morse family of hypersurfaces $h_{v}^{-1}(0)_{v \in \Lambda_{0}}$.

Proof. For any $\boldsymbol{v}=\left(v_{1}, v_{2}, v_{3}, v_{4}\right) \in \Lambda_{0}$, we have $\boldsymbol{v} \neq \mathbf{0}$. Without loss of the generality, we might assume that $v_{1}>0$, then $v_{1}=\sqrt{v_{3}^{2}+v_{4}^{2}-v_{2}^{2}}$. So that

$$
H(u, \boldsymbol{v})=-x_{1}(u) \sqrt{1+v_{3}^{2}+v_{4}^{2}-v_{2}^{2}}-x_{2}(u) v_{2}+x_{3}(u) v_{3}+x_{4}(u) v_{4}+1,
$$

where $\boldsymbol{X}(u)=\left(x_{1}(u), x_{2}(u), x_{3}(u), x_{4}(u)\right)$. We have to prove the mapping

$$
\Delta^{*} H=\left(H, \frac{\partial H}{\partial u_{1}}, \frac{\partial H}{\partial u_{2}}\right)
$$

is non-singular at any point. The Jacobian matrix of $\Delta^{*} H$ is given as follows:

$$
\left(\begin{array}{ccccc}
\left\langle\boldsymbol{X}_{u_{1}}, \boldsymbol{v}\right\rangle & \left\langle\boldsymbol{X}_{u_{2}}, \boldsymbol{v}\right\rangle & x_{1} \frac{v_{2}}{v_{1}}-x_{2} & -x_{1} \frac{v_{3}}{v_{1}}+x_{3} & -x_{1} \frac{v_{4}}{v_{1}}+x_{4} \\
\left\langle\boldsymbol{X}_{u_{1} u_{1}}, \boldsymbol{v}\right\rangle & \left\langle\boldsymbol{X}_{u_{1} u_{2}}, \boldsymbol{v}\right\rangle & x_{1 u_{1}} \frac{v_{2}}{v_{1}}-x_{2 u_{1}} & -x_{1 u_{1}} \frac{v_{3}}{v_{1}}+x_{3 u_{1}} & -x_{1 u_{1}} \frac{v_{4}}{v_{1}}+x_{4 u_{1}} \\
\left\langle\boldsymbol{X}_{u_{2} u_{1}}, \boldsymbol{v}\right\rangle & \left\langle\boldsymbol{X}_{u_{2} u_{2}}, \boldsymbol{v}\right\rangle & x_{1 u_{2}} \frac{v_{2}}{v_{1}}-x_{2 u_{2}} & -x_{1 u_{2}} \frac{v_{3}}{v_{1}}+x_{3 u_{2}} & -x_{1 u_{2}} \frac{v_{4}}{v_{1}}+x_{4 u_{2}}
\end{array}\right) .
$$

We claim that it will suffice to show that the determinant of the matrix

$$
A=\left(\begin{array}{ccc}
x_{1} \frac{v_{2}}{v_{1}}-x_{2} & -x_{1} \frac{v_{3}}{v_{1}}+x_{3} & -x_{1} \frac{v_{4}}{v_{1}}+x_{4} \\
x_{1 u_{1}} \frac{v_{2}}{v_{1}}-x_{2 u_{1}} & -x_{1 u_{1}} \frac{v_{3}}{v_{1}}+x_{3 u_{1}} & -x_{1 u_{1}} \frac{v_{4}}{v_{1}}+x_{4 u_{1}} \\
x_{1 u_{2}} \frac{v_{2}}{v_{1}}-x_{2 u_{2}} & -x_{1 u_{2}} \frac{v_{3}}{v_{1}}+x_{3 u_{2}} & -x_{1 u_{2}} \frac{v_{4}}{v_{1}}+x_{4 u_{2}}
\end{array}\right),
$$


does not vanish at $(u, \boldsymbol{v}) \in \Delta^{*} H^{-1}(\mathbf{0})$. In this case, $\boldsymbol{v}=\mathbb{G}_{n}^{ \pm}(u)$ and we denote

$$
\boldsymbol{b}_{1}=\left(\begin{array}{c}
x_{1} \\
x_{1 u_{1}} \\
x_{1 u_{2}}
\end{array}\right), \boldsymbol{b}_{2}=\left(\begin{array}{c}
x_{2} \\
x_{2 u_{1}} \\
x_{2 u_{2}}
\end{array}\right), \boldsymbol{b}_{3}=\left(\begin{array}{c}
x_{3} \\
x_{3 u_{1}} \\
x_{3 u_{2}}
\end{array}\right), \boldsymbol{b}_{4}=\left(\begin{array}{c}
x_{4} \\
x_{4 u_{1}} \\
x_{4 u_{2}}
\end{array}\right) .
$$

Then we have

$$
\operatorname{det} A=-\frac{v_{1}}{v_{1}} \operatorname{det}\left(\boldsymbol{b}_{2}, \boldsymbol{b}_{3}, \boldsymbol{b}_{4}\right)+\frac{v_{2}}{v_{1}} \operatorname{det}\left(\boldsymbol{b}_{1}, \boldsymbol{b}_{3}, \boldsymbol{b}_{4}\right)-\frac{v_{3}}{v_{1}} \operatorname{det}\left(\boldsymbol{b}_{1}, \boldsymbol{b}_{2}, \boldsymbol{b}_{4}\right)+\frac{v_{4}}{v_{1}} \operatorname{det}\left(\boldsymbol{b}_{1}, \boldsymbol{b}_{2}, \boldsymbol{b}_{3}\right) .
$$

On the other hand, we have

$$
\boldsymbol{X} \wedge \boldsymbol{X}_{u_{1}} \wedge \boldsymbol{X}_{u_{2}}=\left(-\operatorname{det}\left(\boldsymbol{b}_{2}, \boldsymbol{b}_{3}, \boldsymbol{b}_{4}\right), \operatorname{det}\left(\boldsymbol{b}_{1}, \boldsymbol{b}_{3}, \boldsymbol{b}_{4}\right), \operatorname{det}\left(\boldsymbol{b}_{1}, \boldsymbol{b}_{2}, \boldsymbol{b}_{4}\right),-\operatorname{det}\left(\boldsymbol{b}_{1}, \boldsymbol{b}_{2}, \boldsymbol{b}_{3}\right)\right) \text {. }
$$

Therefore we have

$$
\begin{aligned}
\operatorname{det} A & =\left\langle\left(-\frac{v_{1}}{v_{1}},-\frac{v_{2}}{v_{1}},-\frac{v_{3}}{v_{1}},-\frac{v_{4}}{v_{1}}\right), \boldsymbol{X} \wedge \boldsymbol{X}_{u_{1}} \wedge \boldsymbol{X}_{u_{2}}\right\rangle \\
& =-\frac{1}{v_{1}}\left\langle\mathbb{G}_{n}^{ \pm},\left\|\boldsymbol{X} \wedge \boldsymbol{X}_{u_{1}} \wedge \boldsymbol{X}_{u_{2}}\right\| \boldsymbol{N}\right\rangle \\
& =\mp \frac{\left\|\boldsymbol{X} \wedge \boldsymbol{X}_{u_{1}} \wedge \boldsymbol{X}_{u_{2}}\right\|}{v_{1}} \neq 0 .
\end{aligned}
$$

Let $\boldsymbol{X}: U \longrightarrow H_{1}^{3}$ be a timelike surface in $H_{1}^{3}$ and $\mathbb{G}_{n}^{ \pm}$be the AdS-nullcone Gauss image on $M=\boldsymbol{X}(U)$. We denote $\boldsymbol{X}(u)=\left(x_{1}(u), x_{2}(u), x_{3}(u), x_{4}(u)\right)$ and $\mathbb{G}_{n}^{ \pm}(u)=\left(v_{1}(u), v_{2}(u), v_{3}(u), v_{4}(u)\right)$ as coordinate representations. We define a smooth mapping

$$
\mathcal{G}^{ \pm}: U \longrightarrow P T^{*}\left(\Lambda_{0}\right)
$$

by $\mathcal{G}^{ \pm}(u)=\left(\mathbb{G}_{n}^{ \pm}(u),\left[\left(x_{1} v_{2}-x_{2} v_{1}\right):\left(-x_{1} v_{3}+x_{3} v_{1}\right):\left(-x_{1} v_{4}+x_{4} v_{1}\right)\right]\right)$. Then by the above proposition we have the following corollary.

Corollary 3.4 For any timelike surface $\boldsymbol{X}: U \longrightarrow H_{1}^{3}$, the AdS-null height function $H$ : $U \times \Lambda_{0} \longrightarrow \mathbb{R}$ on $\boldsymbol{X}$ is a generating family of the Legendrian embedding $\mathcal{G}^{ \pm}$.

Therefore we conclude that the AdS-nullcone Gauss image $\mathbb{G}_{n}^{ \pm}$can be regarded as a Legendrian map and $\mathbb{G}_{n}^{ \pm}(U)$ can be regarded as a wave front set of $\mathcal{G}^{ \pm}$.

\section{The AdS-torus cylindrical pedals of timelike surfaces}

In this section we consider a surface associated to $M=\boldsymbol{X}(U)$, whose singular set is diffeomophic to that of AdS-nullcone Gauss image. We can use this surface to investigate the relationship between the AdS-nullcone Gauss image $\mathbb{G}_{n}^{ \pm}$and the AdS-torus Gauss map $\widetilde{\mathbb{G}_{n}^{ \pm}}$of a timelike surface in the Anti de Sitter 3-space. For any timelike surface $\boldsymbol{X}: U \longrightarrow H_{1}^{3}$, we define a smooth mapping $A C P_{M}: U \longrightarrow T_{1}^{2} \times \mathbb{R}^{*}$ by

$$
A C P_{M}(u)=\left(\widetilde{\mathbb{G}_{n}^{ \pm}}(u),-\left\langle\boldsymbol{X}(u), \widetilde{\mathbb{G}_{n}^{ \pm}}(u)\right\rangle\right)=\left(\widetilde{\mathbb{G}_{n}^{ \pm}}(u), \frac{1}{\xi(u)}\right) .
$$

We call it the AdS-torus cylindrical pedal of $M=\boldsymbol{X}(U)$, where $\mathbb{R}^{*}=\mathbb{R} \backslash\{0\}$. We define a diffeomorphism $\phi: T_{1}^{2} \times \mathbb{R}^{*} \longrightarrow \Lambda_{0}$ by $\phi(\boldsymbol{v}, \lambda)=\lambda^{-1} \boldsymbol{v}$. It is easy to check that $\phi\left(A C P_{M}(u)\right)=$ $\mathbb{G}_{n}^{ \pm}(u)$, this means that the singular sets of $\mathbb{G}_{n}^{ \pm}$and $A C P_{M}$ are diffeomorphic.

We now consider a family functions $\bar{H}: U \times T_{1}^{2} \times \mathbb{R}^{*} \longrightarrow \mathbb{R}$ defined by

$$
\bar{H}(u, \boldsymbol{v}, \lambda)=\langle\boldsymbol{X}(u), \boldsymbol{v}\rangle+\lambda=\widetilde{H}(u, \boldsymbol{v})+\lambda,
$$

we call it the extended AdS-torus height function on $M=\boldsymbol{X}(U)$. By the similar calculation to the proof of Proposition 3.1(1), we have

$$
\mathcal{D}_{\bar{H}}=\left\{\left(\widetilde{\mathbb{G}_{n}^{ \pm}}(u), \frac{1}{\xi(u)}\right) \mid u \in U\right\}=\left\{A C P_{M}(u) \mid u \in U\right\} .
$$


On the other hand, we consider the canonical projection $\pi_{1}: T_{1}^{2} \times \mathbb{R}^{*} \longrightarrow T_{1}^{2}$. Then we have $\pi_{1} \mid \mathcal{D}_{\bar{H}}$ can be identified with the AdS-torus Gauss map $\widetilde{\mathbb{G}_{n}^{ \pm}}$of X. Since

$$
\mathbb{G}_{n}^{ \pm}(u)=-\frac{1}{\left\langle\boldsymbol{X}(u), \widetilde{\mathbb{G}_{n}^{ \pm}}(u)\right\rangle} \widetilde{\mathbb{G}_{n}^{ \pm}}(u)=\xi(u) \widetilde{\mathbb{G}_{n}^{ \pm}}(u),
$$

we have $\phi\left(\mathcal{D}_{\bar{H}}\right)=\left\{\mathbb{G}_{n}^{ \pm}(u) \mid u \in U\right\}=\mathcal{D}_{H}$. Therefore, we may say that the AdS-nullcone Gauss image $\mathbb{G}_{n}^{ \pm}$is a lift of the AdS-torus Gauss map $\widetilde{\mathbb{G}_{n}^{ \pm}}$. In fact, we also have

$$
\Sigma_{*}(\bar{H})=\left\{\left(u, \widetilde{\mathbb{G}_{n}^{ \pm}}(u),-\left\langle\boldsymbol{X}(u), \widetilde{\mathbb{G}_{n}^{ \pm}}(u)\right\rangle\right) \mid u \in U\right\} .
$$

We remark that similar discussions apply to the extended AdS-torus height function $\bar{H}$ and AdS-torus height function $\widetilde{H}$, and it follows that we have $\bar{H}$ and $\widetilde{H}$ are Morse families.

On the other hand, for any $\boldsymbol{v}=\left(v_{1}, v_{2}, v_{3}, v_{4}\right) \in T_{1}^{2}$, we consider a coordinate neighborhood $U_{24}^{+}=\left\{\boldsymbol{v}=\left(v_{1}, v_{2}, v_{3}, v_{4}\right) \in T_{1}^{2} \mid v_{2}>0\right.$ and $\left.v_{4}>0\right\}$, then

$$
\bar{H}(u, \boldsymbol{v}, \lambda)=\widetilde{H}(u, \boldsymbol{v})+\lambda=-x_{1} v_{1}-x_{2} \sqrt{1-v_{1}^{2}}+x_{3} v_{3}+x_{4} \sqrt{1-v_{3}^{2}}+\lambda .
$$

We now consider smooth mappings $\mathcal{L}_{\bar{H}}:{\widetilde{\mathbb{G}_{n}^{ \pm}}}^{-1}\left(U_{24}^{+}\right) \longrightarrow T^{*}\left(T_{1}^{2}\right) \times \mathbb{R}^{*}$ defined by

$$
\mathcal{L}_{\bar{H}}(u)=\left(\widetilde{\mathbb{G}_{n}^{ \pm}}(u),\left[\frac{\partial \bar{H}}{\partial v_{1}}: \frac{\partial \bar{H}}{\partial v_{3}}: \frac{\partial \bar{H}}{\partial \lambda}\right], \frac{1}{\xi(u)}\right)=\left(\widetilde{\mathbb{G}_{n}^{ \pm}}(u), \frac{\partial \bar{H}}{\partial v_{1}}, \frac{\partial \bar{H}}{\partial v_{3}}, \frac{1}{\xi(u)}\right)
$$

and $L_{\widetilde{H}}:{\widetilde{\mathbb{G}_{n}^{ \pm}}}^{-1}\left(U_{24}^{+}\right) \longrightarrow T^{*}\left(T_{1}^{2}\right)$ defined by

$$
L_{\widetilde{H}}(u)=\left(\widetilde{\mathbb{G}_{n}^{ \pm}}(u), \frac{\partial \widetilde{H}}{\partial v_{1}}, \frac{\partial \widetilde{H}}{\partial v_{3}}\right)=\left(\widetilde{\mathbb{G}_{n}^{ \pm}}(u), \frac{\partial \bar{H}}{\partial v_{1}}, \frac{\partial \bar{H}}{\partial v_{3}}\right) .
$$

According to these definitions we have $\mathcal{L}_{\bar{H}}$ is a Legendrian embedding whose generating family is the extended AdS-torus height function $\bar{H}$ and $L_{\widetilde{H}}$ is a Lagrangian embedding whose generating family is the AdS-torus height function $\widetilde{H}$. The details on Lagrangian singularities can be found in $[1,32]$. We now consider the canonical projection

$$
\pi: T^{*}\left(T_{1}^{2}\right) \times \mathbb{R}^{*} \longrightarrow T^{*}\left(T_{1}^{2}\right), \pi(\boldsymbol{v}, \lambda)=\boldsymbol{v}
$$

then $\pi\left(\mathcal{L}_{\bar{H}}\right)=L_{\widetilde{H}}$. We remark that if we adopt other local coordinates on $T_{1}^{2}$, exactly the same results hold. Therefore we have the following proposition.

Proposition 4.1 Under the same assumptions as in the above arguments, we have the following:

(1) The AdS-torus Gauss map $\widetilde{\mathbb{G}_{n}^{ \pm}}$is a Lagrangian map. The corresponding Lagrangian embedding $L_{\widetilde{H}}$ is called the Lagrangian lift of the AdS-torus Gauss map $\widetilde{\mathbb{G}_{n}^{ \pm}}$;

(2) The Legendrian lift $\mathcal{G}^{ \pm}$of the AdS-nullcone Gauss image $\mathbb{G}_{n}^{ \pm}$is a covering of the Lagrangian lift $L_{\widetilde{H}}$ of the AdS-torus Gauss map $\widetilde{\mathbb{G}_{n}^{ \pm}}$.

Proof. The assertion (1) follows from the above arguments.

On the other hand, for any $\boldsymbol{v} \in T_{1}^{2}$, without loss of the generality, we can assume that $v_{2}>0$ and $v_{4}>0$. Then we have $v_{2}=\sqrt{1-v_{1}^{2}}, v_{4}=\sqrt{1-v_{3}^{2}}$, so we can regard $\left(v_{1}, v_{3}\right)$ as the coordinate system of $T_{1}^{2}$. Therefore, the homogeneous coordinates of $P T^{*}\left(T_{1}^{2} \times \mathbb{R}^{*}\right)$ can be expressed as $\left(v_{1}, v_{3}, \lambda,\left[\varsigma_{1}: \varsigma_{2}: \varsigma\right]\right)$. Moreover, if $\varsigma \neq 0$, we have

$$
\left(v_{1}, v_{3}, \lambda,\left[\varsigma_{1}: \varsigma_{2}: \varsigma\right]\right)=\left(v_{1}, v_{3}, \lambda,\left[\frac{\varsigma_{1}}{\varsigma}: \frac{\varsigma_{2}}{\varsigma}: 1\right]\right)
$$


so that we can adopt the corresponding affine coordinates $\left(v_{1}, v_{3}, \lambda, \rho_{1}, \rho_{2}\right)$, where $\rho_{i}=\varsigma_{i} / \varsigma$. By the above argument we can naturally regard $T^{*}\left(T_{1}^{2}\right) \times \mathbb{R}^{*}$ as the affine part of $P T^{*}\left(T_{1}^{2} \times \mathbb{R}^{*}\right)$. We also have the following relation:

$$
H \circ\left(i d_{U} \times \phi\right)(u, \boldsymbol{v}, \lambda)=H\left(u, \lambda^{-1} \boldsymbol{v}\right)=\lambda^{-1} \bar{H}(u, \boldsymbol{v}, \lambda) .
$$

This means that $H \circ\left(i d_{U} \times \phi\right)$ and $\bar{H}$ are $\mathcal{C}$-equivalent in the sense of Mather[18]. So that these generating families correspond to the same Legendrian submanifold (cf., [1,32]. Then we have a unique contact diffeomorphism $\Phi: P T^{*}\left(T_{1}^{2} \times \mathbb{R}^{*}\right) \longrightarrow P T^{*} \Lambda_{0}$ covering $\phi: T_{1}^{2} \times \mathbb{R}^{*} \longrightarrow \Lambda_{0}$ such that $\Phi \circ \mathcal{L}_{\bar{H}}=\mathcal{G}^{ \pm}$. Therefore, $\mathcal{G}^{ \pm}$is a covering of $L_{\widetilde{H}}$.

\section{Contact with AdS-horospheres}

In this section we consider the geometric meaning of the singularities of the AdS-nullcone Gauss image of a timelike surface $M=\boldsymbol{X}(U)$ in $H_{1}^{3}$. We consider the contact of timelike surfaces with AdS-horospheres in the sense of Montaldi [24]. Let $X_{i}, Y_{i}(i=1,2)$ be submanifolds of $\mathbb{R}^{n}$ with $\operatorname{dim} X_{1}=\operatorname{dim} X_{2}$ and $\operatorname{dim} Y_{1}=\operatorname{dim} Y_{2}$. We say that the contact of $X_{1}$ and $Y_{1}$ at $y_{1}$ is the same type as the contact of $X_{2}$ and $Y_{2}$ at $y_{2}$ if there is a diffeomorphism germ $\Phi:\left(\mathbb{R}^{n}, y_{1}\right) \longrightarrow\left(\mathbb{R}^{n}, y_{2}\right)$ such that $\Phi\left(X_{1}\right)=X_{2}$ and $\Phi\left(Y_{1}\right)=Y_{2}$. In this case we write $K\left(X_{1}, Y_{1} ; y_{1}\right)=K\left(X_{2}, Y_{2} ; y_{2}\right)$. It is clear that in the definition $\mathbb{R}^{n}$ could be replaced by any manifold. In his paper [24], Montaldi gives a characterization of the notion of contact by using the terminology of singularity theory.

Theorem 5.1 Let $X_{i}, Y_{i}(i=1,2)$ be submanifolds of $\mathbb{R}^{n}$ with $\operatorname{dim} X_{1}=\operatorname{dim} X_{2}$ and $\operatorname{dim} Y_{1}=$ $\operatorname{dim} Y_{2}$. Let $g_{i}:\left(X_{i}, x_{i}\right) \longrightarrow\left(\mathbb{R}^{n}, y_{i}\right)$ be immersion germs and $f_{i}:\left(\mathbb{R}^{n}, y_{i}\right) \longrightarrow\left(\mathbb{R}^{p}, \mathbf{0}\right)$ be submersion germs with $\left(Y_{i}, y_{i}\right)=\left(f_{i}^{-1}(\mathbf{0}), y_{i}\right)$. Then $K\left(X_{1}, Y_{1} ; y_{1}\right)=K\left(X_{2}, Y_{2} ; y_{2}\right)$ if and only if $f_{1} \circ g_{1}$ and $f_{2} \circ g_{2}$ are $\mathcal{K}$-equivalent.

For the definition of the $\mathcal{K}$-equivalence, See Martinet [19]. We now consider a function $\mathcal{H}: H_{1}^{3} \times \Lambda_{0} \longrightarrow \mathbb{R}$ defined by $\mathcal{H}(\boldsymbol{u}, \boldsymbol{v})=\langle\boldsymbol{u}, \boldsymbol{v}\rangle+1$. For any $\boldsymbol{v}_{0} \in \Lambda_{0}$, we denote $\mathfrak{h}_{v_{0}}(\boldsymbol{u})=\mathcal{H}\left(\boldsymbol{u}, \boldsymbol{v}_{0}\right)$ and we define the AdS-horosphere by $\mathfrak{h}_{v_{0}}^{-1}(0)=H_{1}^{3} \cap H P\left(\boldsymbol{v}_{0},-1\right)$. We write $A H\left(\boldsymbol{v}_{0},-1\right)=$ $H_{1}^{3} \cap H P\left(\boldsymbol{v}_{0},-1\right)$. For any $u_{0} \in U$, we consider the null vector $\boldsymbol{v}_{0}^{ \pm}=\mathbb{G}_{n}^{ \pm}\left(u_{0}\right)$. Then we have

$$
\mathfrak{h}_{v_{0}^{ \pm}} \circ \boldsymbol{X}\left(u_{0}\right)=\mathcal{H} \circ\left(\boldsymbol{X} \times i d_{\Lambda_{0}}\right)\left(u_{0}, \boldsymbol{v}_{0}\right)=H\left(u_{0}, \mathbb{G}_{n}^{ \pm}\left(u_{0}\right)\right)=0 .
$$

We also have relations

$$
\frac{\partial \mathfrak{h}_{v_{0}^{ \pm}} \circ \boldsymbol{X}}{\partial u_{i}}\left(u_{0}\right)=\frac{\partial H}{\partial u_{i}}\left(u_{0}, \mathbb{G}_{n}^{ \pm}\left(u_{0}\right)\right)=0,
$$

for $i=1,2$. This means that the AdS-horosphere $A H\left(\boldsymbol{v}_{0}^{ \pm},-1\right)$ is tangent to $M=\boldsymbol{X}(U)$ at $p=\boldsymbol{X}\left(u_{0}\right)$. In this case, we call $A H\left(\boldsymbol{v}_{0}^{ \pm},-1\right)$ the tangent $A d S$-horosphere of $M=\boldsymbol{X}(U)$ at $p=\boldsymbol{X}\left(u_{0}\right)$ (or, $\left.u_{0}\right)$, which we write $A H^{ \pm}\left(\boldsymbol{X}, u_{0}\right)$. Let $\boldsymbol{v}_{1}, \boldsymbol{v}_{2}$ be null vectors. If $\boldsymbol{v}_{1}$ and $\boldsymbol{v}_{2}$ are linearly dependent, then $H P\left(\boldsymbol{v}_{1},-1\right)$ and $H P\left(\boldsymbol{v}_{2},-1\right)$ are parallel. Therefore, we say that AdS-horospheres $A H\left(\boldsymbol{v}_{1},-1\right)$ and $A H\left(\boldsymbol{v}_{2},-1\right)$ are parallel, if $\boldsymbol{v}_{1}$ and $\boldsymbol{v}_{2}$ are linearly dependent. Then we have the following lemma.

Lemma 5.2 Let $\boldsymbol{X}: U \longrightarrow H_{1}^{3}$ be a timelike surface. Consider two points $u_{1}, u_{2} \in U$. Then we have the following assertions:

(1) $\mathbb{G}_{n}^{ \pm}\left(u_{1}\right)=\mathbb{G}_{n}^{ \pm}\left(u_{2}\right)$ if and only if $\mathrm{AH}^{ \pm}\left(\boldsymbol{X}, u_{1}\right)=\mathrm{AH}^{ \pm}\left(\boldsymbol{X}, u_{2}\right)$.

(2) $\widetilde{\mathbb{G}_{n}^{ \pm}}\left(u_{1}\right)=\widetilde{\mathbb{G}_{n}^{ \pm}}\left(u_{2}\right)$ if and only if $\operatorname{AH}^{ \pm}\left(\boldsymbol{X}, u_{1}\right)$ and $\mathrm{AH}^{ \pm}\left(\boldsymbol{X}, u_{2}\right)$ are parallel. 
We now consider the contact of $M$ with tangent AdS-horosphere at $p \in M$ as an application of Legendrian singularity theory. The main result in the theory of Legendrian singularities $[1,32]$ is the following:

Theorem 5.3 Let $F, G:\left(\mathbb{R}^{k} \times \mathbb{R}^{n}, \mathbf{0}\right) \longrightarrow(\mathbb{R}, 0)$ be Morse families. Then

(1) $\mathcal{L}_{F}$ and $\mathcal{L}_{G}$ are Legendrian equivalent if and only if $F$ and $G$ are $P-\mathcal{K}$ - equivalent;

(2) $\mathcal{L}_{F}$ is Legendrian stable if and only if $F$ is a $\mathcal{K}$-versal deformation of $f=F \mid \mathbb{R}^{k} \times\{\mathbf{0}\}$.

For the definitions of the Legendrian equivalence, Legendrian stable, $P-\mathcal{K}$-equivalence and $\mathcal{K}$-versal deformation, See $[1,19,32]$.

Let $\mathbb{G}_{n i}^{ \pm}:\left(U, u_{i}\right) \longrightarrow\left(\Lambda_{0}, \boldsymbol{v}_{i}^{ \pm}\right)$(for $\left.i=1,2\right)$ be AdS-nullcone Gauss image germs of timelike surface germs $\boldsymbol{X}_{i}:\left(U, u_{i}\right) \longrightarrow\left(H_{1}^{3}, \boldsymbol{X}_{i}\left(u_{i}\right)\right)$. We say that $\mathbb{G}_{n 1}^{ \pm}$and $\mathbb{G}_{n 2}^{ \pm}$are $\mathcal{A}$-equivalent if there exist diffeomorphism germs $\phi:\left(U, u_{1}\right) \longrightarrow\left(U, u_{2}\right)$ and $\Phi:\left(H_{1}^{3}, \boldsymbol{v}_{1}^{ \pm}\right) \longrightarrow\left(H_{1}^{3}, \boldsymbol{v}_{2}^{ \pm}\right)$such that $\Phi \circ \mathbb{G}_{n 1}^{ \pm}=\mathbb{G}_{n_{2}}^{ \pm} \circ \phi$. Suppose the regular set of $\mathbb{G}_{n i}^{ \pm}$is dense in $\left(U, u_{i}\right)$ for each $i=1,2$. It follows from Proposition A.2 in the appendix of [11] (See also [33]) that $\mathbb{G}_{n 1}^{ \pm}$and $\mathbb{G}_{n 2}^{ \pm}$are $\mathcal{A}$-equivalent if and only if the corresponding Legendrian embedding germs $\mathcal{G}_{1}^{ \pm}:\left(U, u_{1}\right) \longrightarrow\left(\Delta_{1}, \mathbf{z}_{1}\right)$ and $\mathcal{G}_{2}^{ \pm}:\left(U, u_{2}\right) \longrightarrow\left(\Delta_{1}, \mathbf{z}_{2}\right)$ are Legendrian equivalent. This condition is also equivalent to the condition that two generating families $H_{1}$ and $H_{2}$ are $P-\mathcal{K}$-equivalent by Theorem 5.3. Here, $H_{i}:\left(U \times \Lambda_{0},\left(u_{i}, \boldsymbol{v}_{i}^{ \pm}\right)\right) \longrightarrow \mathbb{R}$ is the corresponding AdS-null height function germ of $\boldsymbol{X}_{i}$.

On the other hand, we denote $h_{i, v_{i}^{ \pm}}=H_{i}\left(u, \boldsymbol{v}_{i}^{ \pm}\right)$; then we have $h_{i, v_{i}^{ \pm}}(u)=\mathfrak{h}_{v_{i}^{ \pm}} \circ \boldsymbol{X}_{i}(u)$. By Theorem 5.1,

$$
K\left(\boldsymbol{X}_{1}(U), \mathrm{AH}^{ \pm}\left(\boldsymbol{X}_{1}, u_{1}\right), \boldsymbol{v}_{1}^{ \pm}\right)=K\left(\boldsymbol{X}_{2}(U), \mathrm{AH}^{ \pm}\left(\boldsymbol{X}_{2}, u_{2}\right), \boldsymbol{v}_{2}^{ \pm}\right)
$$

if and only if $h_{1, v_{1}^{ \pm}}$and $h_{2, v_{2}^{ \pm}}$are $\mathcal{K}$-equivalent. Therefore, we can apply the above arguments to our situation. We denote by $Q^{ \pm}\left(\boldsymbol{X}, u_{0}\right)$ the local ring of the function germ $h_{v_{0}^{ \pm}}:\left(U, u_{0}\right) \longrightarrow \mathbb{R}$, where $\boldsymbol{v}_{0}^{ \pm}=\mathbb{G}_{n}^{ \pm}\left(u_{0}\right)$. We remark that we can write the local ring explicitly as follows:

$$
Q^{ \pm}\left(\boldsymbol{X}, u_{0}\right)=\frac{C_{u_{0}}^{\infty}(U)}{\left\langle\left\langle\boldsymbol{X}(u), \mathbb{G}_{n}^{ \pm}\left(u_{0}\right)\right\rangle+1\right\rangle_{C_{u_{0}}(U)}},
$$

where $C_{u_{0}}^{\infty}(U)$ is the local ring of function germs at $u_{0}$ with the unique maximal ideal $\mathcal{M}_{u_{0}}(U)$.

Theorem 5.4 Let $\boldsymbol{X}_{i}:\left(U, u_{i}\right) \longrightarrow\left(H_{1}^{3}, \boldsymbol{X}_{i}\left(u_{i}\right)\right)$ (for $\left.i=1,2\right)$ be timelike surface germs such that the corresponding Legendrian embedding germs $\mathcal{G}_{i}^{ \pm}:\left(U, u_{i}\right) \longrightarrow\left(\Delta_{1}, \boldsymbol{z}_{i}\right)$ are Legendrian stable. Then the following conditions are equivalent:

(1) AdS-nullcone Gauss image germs $\mathbb{G}_{n_{1}}^{ \pm}$and $\mathbb{G}_{n_{2}}^{ \pm}$are $\mathcal{A}$-equivalent;

(2) $H_{1}$ and $H_{2}$ are $P$ - $\mathcal{K}$-equivalent;

(3) $h_{1, v_{1}^{ \pm}}$and $h_{2, v_{2}^{ \pm}}$are $\mathcal{K}$-equivalent;

(4) $K\left(\boldsymbol{X}_{1}(U), A H^{ \pm}\left(\boldsymbol{X}_{1}, u_{1}\right), \boldsymbol{v}_{1}^{ \pm}\right)=K\left(\boldsymbol{X}_{2}(U), A H^{ \pm}\left(\boldsymbol{X}_{2}, u_{2}\right), \boldsymbol{v}_{2}^{ \pm}\right)$;

(5) $Q^{ \pm}\left(\boldsymbol{X}_{1}, u_{1}\right)$ and $Q^{ \pm}\left(\boldsymbol{X}_{2}, u_{2}\right)$ are isomorphic as $\mathbb{R}$-algebras.

For a timelike surface germ $\boldsymbol{X}:\left(U, u_{0}\right) \longrightarrow\left(H_{1}^{3}, \boldsymbol{X}\left(u_{0}\right)\right)$, we call $\boldsymbol{X}^{-1}\left(A H\left(\mathbb{G}_{n}^{ \pm}\left(u_{0}\right),-1\right), u_{0}\right)$ the tangent Anti de Sitter horospherical indicatrix germ (briefly, tangent AdS-horospherical indicatrix germ) of $\boldsymbol{X}$. In general we have the following proposition:

Proposition 5.5 Let $\boldsymbol{X}_{i}:\left(U, u_{i}\right) \longrightarrow\left(H_{1}^{3}, \boldsymbol{X}_{i}\left(u_{i}\right)\right)$ (for $\left.i=1,2\right)$ be timelike surface germs such that their AdSh $h^{ \pm}$-parabolic sets have no interior points as subspaces of $U$. If AdS-nullcone Gauss image germs $\mathbb{G}_{n 1}^{ \pm}$and $\mathbb{G}_{n 2}^{ \pm}$are $\mathcal{A}$-equivalent, then

$$
K\left(\boldsymbol{X}_{1}(U), A H^{ \pm}\left(\boldsymbol{X}_{1}, u_{1}\right), \boldsymbol{v}_{1}^{ \pm}\right)=K\left(\boldsymbol{X}_{2}(U), A H^{ \pm}\left(\boldsymbol{X}_{2}, u_{2}\right), \boldsymbol{v}_{2}^{ \pm}\right) .
$$


In this case, $\boldsymbol{X}_{1}^{-1}\left(A H\left(\mathbb{G}_{n 1}^{ \pm}\left(u_{1}\right),-1\right), u_{1}\right)$ and $\boldsymbol{X}_{2}^{-1}\left(A H\left(\mathbb{G}_{n_{2}}^{ \pm}\left(u_{2}\right),-1\right), u_{2}\right)$ are diffeomorphic as set germs.

From the above proposition, the diffeomorphism type of the tangent AdS-horospherical indicatrix germ is an invariant of $\mathcal{A}$-classification of the AdS-nullcone Gauss image germ of $\boldsymbol{X}$. Moreover, we need some numerical $\mathcal{K}$-invariants for a function germ. We denote

$$
\operatorname{Ah}^{ \pm}-\operatorname{ord}\left(\boldsymbol{X}, u_{0}\right)=\operatorname{dim} \frac{C_{u_{0}}^{\infty}(U)}{\left\langle h_{v_{0}^{ \pm}}\left(u_{0}\right), \partial h_{v_{0}^{ \pm}}\left(u_{0}\right) / \partial u_{i}\right\rangle_{{u_{0}}^{\infty}(U)}},
$$

where $\boldsymbol{v}_{0}^{ \pm}=\mathbb{G}_{n}^{ \pm}\left(u_{0}\right)$. Usually $\operatorname{Ah}^{ \pm}$-ord $\left(\boldsymbol{X}, u_{0}\right)$ is called the $\mathcal{K}$-codimension of $h_{v_{0}^{ \pm}}$. However, We call it the order of contact with tangent $A d S$-horosphere at $\boldsymbol{X}\left(u_{0}\right)$. We also have the notion of corank of function germs:

$$
\operatorname{Ah}^{ \pm}-\operatorname{corank}\left(\boldsymbol{X}, u_{0}\right)=2-\operatorname{rankHess}\left(h_{v_{0}^{ \pm}}\right)\left(u_{0}\right),
$$

By Proposition 3.1, $\boldsymbol{X}\left(u_{0}\right)$ is an $\mathrm{AdSh}^{ \pm}$-parabolic point if and only if $\mathrm{Ah}^{ \pm}$-corank $\left(\boldsymbol{X}, u_{0}\right) \geq$ 1 and $\boldsymbol{X}\left(u_{0}\right)$ is an AdS-horospherical point if and only if $\operatorname{Ah}^{ \pm}$-corank $\left(\boldsymbol{X}, u_{0}\right)=2$. On the other hand, a function germ $f:\left(\mathbb{R}^{n-1}, \boldsymbol{a}\right) \longrightarrow \mathbb{R}$ has the $A_{k}$-singularity if $f$ is $\mathcal{K}$-equivalent to the germ $\pm u_{1}^{2} \pm \cdots \pm u_{n-2}^{2}+u_{n-1}^{k+1}$. If $\operatorname{Ah}^{ \pm}-\operatorname{corank}\left(\boldsymbol{X}, u_{0}\right)=1$, the AdS-null height function $h_{v_{0}^{ \pm}}$ has the $A_{k}$-singularity at $u_{0}$ and is generic. In this case we have $\operatorname{Ah}^{ \pm}-\operatorname{ord}\left(\boldsymbol{X}, u_{0}\right)=k$. This number is equal to the order of contact in the classical sense (cf. [3]). This is the reason why we call $\mathrm{Ah}^{ \pm}-\operatorname{ord}\left(\boldsymbol{X}, u_{0}\right)$ the order of contact with the AdS-horosphere at $\boldsymbol{X}\left(u_{0}\right)$.

\section{Classification of singularities of AdS-nullcone Gauss images}

In this section we give the generic classification of singularities of AdS-nullcone Gauss images. The arguments are almost the same as those of [11], so that we omit the details. We consider the space of timelike embeddings $\operatorname{Emb}_{T}\left(U, H_{1}^{3}\right)$ with the Whitney $C^{\infty}$-topology. By the classification of stable Legendrian singularities of $n=3$ and the transversality theorem of [11] (Proposition 7.1), we have the following theorem.

Theorem 6.1 There exists an open dense subset $\mathcal{O} \subset \operatorname{Emb}_{T}\left(U, H_{1}^{3}\right)$ such that for any $\boldsymbol{X} \in \mathcal{O}$ the following conditions hold.

(1) The AdSh ${ }^{ \pm}$-parabolic set $K_{A d S n}^{ \pm}{ }^{-1}(0)$ is a regular curve. We call such a curve the AdSh ${ }^{ \pm}$parabolic curve.

(2) The AdS-nullcone Gauss image $\mathbb{G}_{n}^{ \pm}$along the AdSh $h^{ \pm}$-parabolic curve is a cuspidal edge except at isolated points. At such the point $\mathbb{G}_{n}^{ \pm}$is the swallowtail.

(3) The cuspidal edge points (swallowtail points) of AdS-nullcone Gauss image $\mathbb{G}_{n}^{ \pm}$correspond to fold points (cusp points) of AdS-torus Gauss map. (cf., Figure 1).
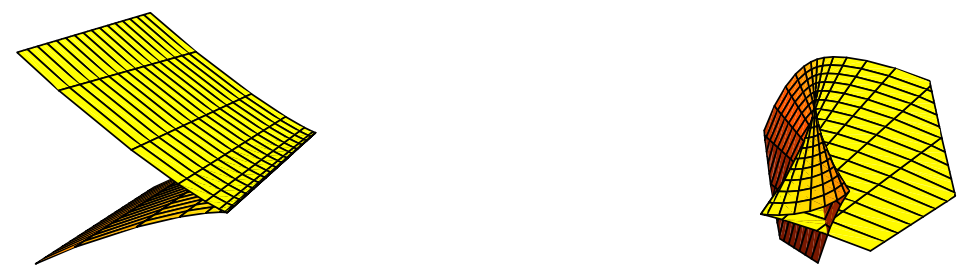

Cuspital edge

Swallowtail

Figure 1 
Following the terminology of Whitney[31], we say that a timelike surface $\boldsymbol{X}: U \longrightarrow H_{1}^{3}$ has the excellent AdS-nullcone Gauss image $\mathbb{G}_{n}^{ \pm}$, the AdS-nullcone Gauss image $\mathbb{G}_{n}^{ \pm}$has only cuspidal edges and swallowtails as singularities.

We now consider the geometric meanings of cuspidal edges and swallowtails of the AdSnullcone Gauss image. We have the following results analogous to the results of [11].

Theorem 6.2 Let $\mathbb{G}_{n}^{ \pm}:\left(U, u_{0}\right) \longrightarrow\left(\Lambda_{0}, \boldsymbol{v}_{0}^{ \pm}\right)$be the excellent AdS-nullcone Gauss image germ of a timelike surface $\boldsymbol{X}$ and $h_{v_{0}}:\left(U, u_{0}\right) \longrightarrow \mathbb{R}$ be the AdS-null height function germ at $u_{0}$, where $\boldsymbol{v}_{0}^{ \pm}=\mathbb{G}_{n}^{ \pm}\left(u_{0}\right)$. Then we have the following.

(1) The point $u_{0}$ is an $A d S h^{ \pm}$-parabolic point of $\boldsymbol{X}$ if and only if $\operatorname{Ah}^{ \pm}$-corank $\left(\boldsymbol{X}, u_{0}\right)=1$.

(2) If $u_{0}$ is an $A d S h^{ \pm}$-parabolic point of $\boldsymbol{X}$, then $h_{v_{0}^{ \pm}}$has the $A_{k}$-singularity for $k=2,3$.

(3) Suppose that $u_{0}$ is an $A d S h^{ \pm}$-parabolic point of $\boldsymbol{X}$. Then the following conditions are equivalent:

(a) $\mathbb{G}_{n}^{ \pm}$has the cuspidal edge at $u_{0}$;

(b) $h_{v_{0}^{ \pm}}$has the $A_{2}$-singularity;

(c) $\mathrm{Ah}^{ \pm}$-order $\left(\boldsymbol{X}, u_{0}\right)=2$;

(d) the tangent AdS-horospherical indicatrix germ is an ordinary cusp, where a curve $C \subset$ $\mathbb{R}^{2}$ is called an ordinary cusp if it is diffeomorphic to the curve given by $\left\{\left(u_{1}, u_{2}\right) \mid u_{1}^{2}-u_{2}^{3}=0\right\}$.

(e) for each $\varepsilon>0$, there exist two points $u_{1}, u_{2} \in U$ such that $\left|u_{0}-u_{i}\right|<\varepsilon$ for $i=1,2$, neither $u_{1}$ nor $u_{2}$ is an AdSh $h^{ \pm}$-parabolic point and the tangent AdS-horospheres to $M=\boldsymbol{X}(U)$ at $u_{1}$ and $u_{2}$ are parallel.

(4) Suppose that $u_{0}$ is an AdSh $h^{ \pm}$-parabolic point of $\boldsymbol{X}$. Then the following conditions are equivalent:

(a) $\mathbb{G}_{n}^{ \pm}$has the swallowtail at $u_{0}$;

(b) $h_{v_{0}^{ \pm}}$has the $A_{3}$-singularity;

(c) $\operatorname{Ah}^{ \pm}$-order $\left(\boldsymbol{X}, u_{0}\right)=3$;

(d) the tangent AdS-horospherical indicatrix germ is a point or a tacnodal, where a curve $C \subset \mathbb{R}^{2}$ is called a tacnodal if it is diffeomorphic to the curve given by $\left\{\left(u_{1}, u_{2}\right) \mid u_{1}^{2}-u_{2}^{4}=0\right\}$.

(e) for each $\varepsilon>0$, there exist three points $u_{1}, u_{2}, u_{3} \in U$ such that $\left|u_{0}-u_{i}\right|<\varepsilon$ for $i=1,2,3$, none of $u_{1}, u_{2}, u_{3}$ are an $A d S h^{ \pm}$-parabolic points and the tangent AdS-horospheres to $M=\boldsymbol{X}(U)$ at $u_{1}, u_{2}$ and $u_{3}$ are parallel.

(f) for each $\varepsilon>0$, there exist two points $u_{1}, u_{2} \in U$ such that $\left|u_{0}-u_{i}\right|<\varepsilon$ for $i=1,2$, neither $u_{1}$ nor $u_{2}$ is an AdS-parabolic point and the tangent AdS-horospheres to $M=\boldsymbol{X}(U)$ at $u_{1}$ and $u_{2}$ are equal.

Proof. By the Proposition 3.1, we have shown that $u_{0}$ is an $\mathrm{AdSh}^{ \pm}$-parabolic point if and only if $\operatorname{Ah}^{ \pm}$-corank $\left(\boldsymbol{X}, u_{0}\right) \geq 1$. Since $n=3$, we have $\operatorname{Ah}^{ \pm}-\operatorname{corank}\left(\boldsymbol{X}, u_{0}\right) \leq 2$. Since the AdS-null height function germ $H:\left(U \times \Lambda_{0},\left(u_{0}, \boldsymbol{v}_{0}^{ \pm}\right)\right) \longrightarrow \mathbb{R}$ can be considered as a generating family of the Legendrian embedding germ $\mathcal{G}^{ \pm}, h_{v_{0}^{ \pm}}$has only the $A_{k}$-singularities $(k=1,2,3)$. This means that the corank of the Hessian matrix of the $h_{v_{0}^{ \pm}}$at an $\mathrm{AdSh}^{ \pm}$-parabolic point is 1 . The assertion (2) also follows. For the same reason, the conditions $(3)\{(a),(b),(c)\}$ (respectively, (4) $\{(a),(b),(c)\})$ are equivalent.

On the other hand, if the AdS-null height function germ $h_{v_{0}^{ \pm}}$has the $A_{2}$-singularity, it is $\mathcal{K}$-equivalent to the germ $\pm u_{1}^{2}+u_{2}^{3}$. Since the $\mathcal{K}$-equivalence preserves the zero level sets, the tangent AdS-horospherical indicatrix germ is diffeomorphic to the curve given by $\pm u_{1}^{2}+u_{2}^{3}=0$. This is the ordinary cusp. The normal form for the $A_{3}$-singularity is given by $\pm u_{1}^{2}+u_{2}^{4}$, so the tangent AdS-horospherical indicatrix germ is diffeomorphic to the curve given by $\pm u_{1}^{2}+u_{2}^{4}=0$. 
This means that the condition $(3)\{(d)\}$ (respectively, $(4)\{(d)\}$ ) is also equivalent to the other conditions.

Suppose that $u_{0}$ is an $\mathrm{AdSh}^{ \pm}$-parabolic point, by Proposition 4.1, the AdS-torus Gauss map has only folds or cusps. If the point $u_{0}$ is a fold point, there is a neighborhood of $u_{0}$ on which the AdS-torus Gauss map is 2 to 1 except the $\mathrm{AdSh}^{ \pm}$-parabolic line (i.e, fold curve). By Lemma 5.2, the condition $(3)(\mathrm{e})$ holds. If the point $u_{0}$ is a cusp, the critical value set is an ordinary cusp. By the normal form, we can understand that the AdS-torus Gauss map is 3 to 1 inside region of the critical value. Moreover, the point $u_{0}$ is in the closure of the region. This means that the condition (4)(e) is satisfied. We can also observe that nearby the cusp point, there are 2 to 1 points which approach to $u_{0}$. However, one of those points is always $\mathrm{AdSh}^{ \pm}$-parabolic point. Since other singularities do not appear in this case, so that the condition (3)(e) (respectively, (4)(e)) characterizes a fold (respectively, a cusp).

For the swallowtail point $u_{0}$, there is a self-intersection curve approaching $u_{0}$. On this curve, there are two distinct points $u_{1}$ and $u_{2}$ such that $\mathbb{G}_{n}^{ \pm}\left(u_{1}\right)=\mathbb{G}_{n}^{ \pm}\left(u_{2}\right)$. By Lemma 5.2, this means that the tangent AdS-horospheres to $M=\boldsymbol{X}(U)$ at $u_{1}$ and $u_{2}$ are equal. Since there are no other singularities in this case, the condition $(4)\{(f)\}$ characterizes a swallowtail point of $\mathbb{G}_{n}^{ \pm}$. This completes the proof.

\section{AdS-null Monge form}

The notion of the Monge form of a surface in Euclidean 3-space is one of the powerful tools for the study of local properties of the surface from the view point of differential geometry. In this section we consider the analogous notion for a timelike surface in $H_{1}^{3}$.

We now consider a function $f\left(u_{1}, u_{2}\right)$ with $f(0)=f_{u_{i}}(0)=0, i=1,2$. Then we have a timelike surface in $H_{1}^{3}$ defined by

$$
\begin{gathered}
\boldsymbol{X}_{f}\left(u_{1}, u_{2}\right)=\left(\sqrt{1+\varepsilon_{1} u_{1}^{2}+\varepsilon_{2} u_{2}^{2}+f^{2}\left(u_{1}, u_{2}\right)}, \frac{\left(1-\varepsilon_{1}\right) u_{1}+\left(1-\varepsilon_{2}\right) u_{2}}{2},\right. \\
\left.f\left(u_{1}, u_{2}\right), \frac{\left(1+\varepsilon_{1}\right) u_{1}+\left(1+\varepsilon_{2}\right) u_{2}}{2}\right),
\end{gathered}
$$

where $\varepsilon_{i}=\operatorname{sign}\left(\boldsymbol{X}_{i}\right)(i=1,2)$. We can easily calculate $\boldsymbol{N}(0)=\left(0,0, \frac{\varepsilon_{2}-\varepsilon_{1}}{2}, 0\right)$, therefore $\mathbb{G}_{n}^{ \pm}(0)=(1,0, \pm 1,0)$. We call $\boldsymbol{X}_{f}$ an Anti de Sitter null Monge form (briefly, AdS-null Monge form). Then we have the following proposition.

Proposition 7.1 Any timelike surface in $H_{1}^{3}$ is locally given by the AdS-null Monge form.

Proof. Let $\boldsymbol{X}: U \longrightarrow H_{1}^{3}$ be a timelike surface. We consider Lorentzian motion of $H_{1}^{3}$ which is a transitive action. Therefore, without loss of the generality, we assume that $p=\boldsymbol{X}(0)=$ $(1,0,0,0)$. We denote $M=\boldsymbol{X}(U)$, we have a basis $\left\{\boldsymbol{X}(0), \boldsymbol{N}(0), \boldsymbol{X}_{u_{1}}(0), \boldsymbol{X}_{u_{2}}(0)\right\}$ of $T_{p} \mathbb{R}_{2}^{4}$ such that $T_{p} M=\left\langle\boldsymbol{X}_{u_{1}}(0), \boldsymbol{X}_{u_{2}}(0)\right\rangle_{\mathbb{R}}$. Applying the Gram-Schmidt procedure we have a pseudoorthonormal basis $\left\{\boldsymbol{X}(0), \boldsymbol{N}(0), \boldsymbol{e}_{1}, \boldsymbol{e}_{2}\right\}$ of $T_{p} \mathbb{R}_{2}^{4}$ such that $T_{p} M=\left\langle\boldsymbol{e}_{1}, \boldsymbol{e}_{2}\right\rangle_{\mathbb{R}}$. In particular, $\left\{\boldsymbol{e}_{1}, \boldsymbol{e}_{2}\right\}$ is an pseudo-orthonormal basis of $T_{p} M$. Since $p=(1,0,0,0), T_{p} M$ is considered to be a subspace of ${ }_{0} \mathbb{R}_{1}^{3}=\left\{\left(0, x_{1}, x_{2}, x_{3}\right) \mid x_{i} \in \mathbb{R}\right\}$. By a rotation of the space ${ }_{0} \mathbb{R}_{1}^{3}$, we might assume that $T_{p} M=\left\{\left(0, \frac{\left(1-\varepsilon_{1}\right) u_{1}+\left(1-\varepsilon_{2}\right) u_{2}}{2}, 0, \frac{\left(1+\varepsilon_{1}\right) u_{1}+\left(1+\varepsilon_{2}\right) u_{2}}{2}\right) \mid u_{i} \in \mathbb{R}\right\} \subset \mathbb{R}_{2}^{4}$. Then the germ $(M, p)$ might be written in the form

$$
\left(f_{0}\left(u_{1}, u_{2}\right), \frac{\left(1-\varepsilon_{1}\right) u_{1}+\left(1-\varepsilon_{2}\right) u_{2}}{2}, f\left(u_{1}, u_{2}\right), \frac{\left(1+\varepsilon_{1}\right) u_{1}+\left(1+\varepsilon_{2}\right) u_{2}}{2}\right)
$$


with function germs $f_{0}\left(u_{1}, u_{2}\right), f\left(u_{1}, u_{2}\right)$. Since $M \subset H_{1}^{3}$, we have the relation

$$
f_{0}\left(u_{1}, u_{2}\right)=\sqrt{1+\varepsilon_{1} u_{1}^{2}+\varepsilon_{2} u_{2}^{2}+f^{2}\left(u_{1}, u_{2}\right)} .
$$

Since we have

$$
T_{p} M=\left\{\left(0, \frac{\left(1-\varepsilon_{1}\right) u_{1}+\left(1-\varepsilon_{2}\right) u_{2}}{2}, 0, \frac{\left(1+\varepsilon_{1}\right) u_{1}+\left(1+\varepsilon_{2}\right) u_{2}}{2}\right) \mid u_{i} \in \mathbb{R}\right\},
$$

the condition $f(0)=0, f_{u_{i}}(0)=0$ are automatically satisfied.

For the null vector $\boldsymbol{v}_{0}^{ \pm}=(1,0, \pm 1,0)$, we consider the AdS-horosphere $\operatorname{AH}\left(\boldsymbol{v}_{0}^{ \pm},-1\right)$. Then we have the AdS-null Monge form of $\operatorname{AH}\left(\boldsymbol{v}_{0}^{ \pm},-1\right)$ :

$\boldsymbol{a}^{ \pm}\left(u_{1}, u_{2}\right)=\left(\frac{\varepsilon_{1} u_{1}^{2}+\varepsilon_{2} u_{2}^{2}}{2}+1, \frac{\left(1-\varepsilon_{1}\right) u_{1}+\left(1-\varepsilon_{2}\right) u_{2}}{2}, \pm \frac{\varepsilon_{1} u_{1}^{2}+\varepsilon_{2} u_{2}^{2}}{2}, \frac{\left(1+\varepsilon_{1}\right) u_{1}+\left(1+\varepsilon_{2}\right) u_{2}}{2}\right)$.

Here, we can easily check the relation $\left\langle\boldsymbol{a}(u), \boldsymbol{v}_{0}^{ \pm}\right\rangle=-1$.

On the other hand, $\boldsymbol{a}^{ \pm}(0)=(1,0,0,0)=p$ and $\boldsymbol{a}_{u_{i}}^{ \pm}(0)$ is equal to the $x_{3+\varepsilon_{i}}$-axis for $i=1,2$. This means that $T_{p} M=T_{p}\left(\boldsymbol{a}^{ \pm}(U)\right)$. Therefore $\boldsymbol{a}^{ \pm}(U)=\mathrm{AH}\left(\boldsymbol{v}_{0}^{ \pm},-1\right)$ is the tangent AdShorosphere of $M=\boldsymbol{X}_{f}(U)$ at $p=\boldsymbol{X}_{f}(0)$. It follows from this fact that the tangent AdShorospherical indicatrix of the AdS-null Monge form germ $\left(\boldsymbol{X}_{f}, 0\right)$ is given as follows:

$$
\boldsymbol{X}_{f}^{-1}\left(A H\left(\boldsymbol{v}_{0}^{ \pm}, 0\right)\right)=\left\{\left(u_{1}, u_{2}\right) \mid \pm 2 f\left(u_{1}, u_{2}\right)=\varepsilon_{1} u_{1}^{2}+\varepsilon_{2} u_{2}^{2}\right\}
$$

On the other hand, since $f(0)=f_{u_{i}}(0)=0$, we may write

$$
f\left(u_{1}, u_{2}\right)=\frac{1}{2} \overline{k_{1}} u_{1}^{2}+\frac{1}{2} \overline{k_{2}} u_{2}^{2}+g\left(u_{1}, u_{2}\right)
$$

where $g \in \mathcal{M}_{2}^{3}$ and $\overline{k_{1}}, \overline{k_{2}}$ are eigenvalues of $\left(f_{u_{i} u_{j}}(0)\right)$. Under this representation, we can easily calculate $\left(\boldsymbol{X}_{f}\right)_{u_{i} u_{j}}(0)=\left(\varepsilon_{i} \delta_{i j}, 0, \bar{k}_{i}, \delta_{i j}, 0\right)$. It follows from this fact that

$$
h_{i j}^{ \pm}(0)=\left\langle\mathbb{G}_{n}^{ \pm}(0),\left(\boldsymbol{X}_{f}\right)_{u_{i} u_{j}}(0)\right\rangle=\varepsilon_{i} \delta_{i j}\left(-1 \pm \bar{k}_{i}\right),
$$

and

$$
g_{i j}(0)=\left\langle\left(\boldsymbol{X}_{f}\right)_{u_{i}}(0),\left(\boldsymbol{X}_{f}\right)_{u_{j}}(0)\right\rangle=\varepsilon_{i} \delta_{i j}
$$

Therefore, we have $k_{i}^{ \pm}(0)=-1 \pm \varepsilon_{i} \bar{k}_{i}$ and

$$
K_{A d S n}^{ \pm}(0)=k_{1}^{ \pm}(0) k_{2}^{ \pm}(0)=\left(-1 \pm \varepsilon_{1} \overline{k_{1}}\right)\left(-1 \pm \varepsilon_{2} \overline{k_{2}}\right) .
$$

The tangent AdS-horospherical indicatrix is given by

$$
\begin{aligned}
\boldsymbol{X}_{f}^{-1}\left(A H\left(\boldsymbol{v}_{0}^{ \pm},-1\right)\right) & =\left\{\left(u_{1}, u_{2}\right) \mid \pm \overline{k_{1}} u_{1}^{2} \pm \overline{k_{2}} u_{2}^{2} \pm 2 g\left(u_{1}, u_{2}\right)-\varepsilon_{1} u_{1}^{2}-\varepsilon_{2} u_{2}^{2}=0\right\} \\
& =\left\{\left(u_{1}, u_{2}\right) \mid \varepsilon_{1} k_{1}^{ \pm}(0) u_{1}^{2}+\varepsilon_{2} k_{2}^{ \pm}(0) u_{2}^{2} \pm 2 g\left(u_{1}, u_{2}\right)=0\right\} .
\end{aligned}
$$

If we try to draw the picture of the AdS-nullcone Gauss image, it might be very hard to give a parameterization. However, by the AdS-null Monge form of the tangent AdS-horospherical indicatrix, we can easy to detect the type of singularities of the AdS-nullcone Gauss image $\mathbb{G}_{n}^{ \pm}$ (or, AdS-torus Gauss map $\widetilde{\mathbb{G}_{n}^{ \pm}}$).

Example 7.1 Consider the function given by

$$
f\left(u_{1}, u_{2}\right)=\frac{1}{2} u_{1}^{2}+u_{2}^{2}+\frac{1}{2} u_{1}^{3} .
$$


Suppose that $\varepsilon_{1}=-1, \varepsilon_{2}=1$ Then $\bar{k}_{1}=1, \bar{k}_{2}=2$. We have $k_{1}^{+}(0)=-2, k_{2}^{+}(0)=1$, $k_{1}^{-}(0)=0, k_{2}^{-}(0)=-3$. So that the origin is an AdSh- parabolic point. The tangent AdShorospherical indicatrix germ at the origin is the ordinary cusp $u_{2}^{2}=-\frac{1}{3} u_{1}^{3}$. By Theorem 6.2, $\mathbb{G}_{n}^{-}\left(\widetilde{\mathbb{G}_{n}^{-}}\right)$is the cuspidal edge (fold) at the origin.

Example 7.2 Consider the function given by

$$
f\left(u_{1}, u_{2}\right)=\frac{1}{2} u_{1}^{2}+\frac{1}{4} u_{2}^{2}+\frac{3}{2} u_{1}^{4} .
$$

Suppose that $\varepsilon_{1}=1, \varepsilon_{2}=-1$ Then $\bar{k}_{1}=1, \bar{k}_{2}=1 / 2$. We have $k_{1}^{+}(0)=0, k_{2}^{+}(0)=-3 / 2$, $k_{1}^{-}(0)=-2, k_{2}^{-}(0)=-1 / 2$. So that the origin is an AdSh $h^{+}$parabolic point. The tangent AdS-horospherical indicatrix germ at the origin is the tacnodal $u_{2}^{2}=u_{1}^{4}$. By Theorem 6.2, $\mathbb{G}_{n}^{-}$ $\left(\widetilde{\mathbb{G}_{n}^{-}}\right)$is the swallowtail (cusp) at the origin.

\section{References}

[1] V. I. Arnol'd, S. M. Gusein-Zade and A. N. Varchenko, Singularities of Differentiable Maps vol. I, Birkhäuser (1986).

[2] Th. Banchoff, T. Gaffney and C. McCrory, Cusps of Gauss mappings. Research Notes in Mathematics, 55. Pitman (Advanced Publishing Program), Boston, Mass.-London (1982).

[3] J. W. Bruce and P. J. Giblin, Curves and singularities (second edition), Cambridge Univ. Press, (1992).

[4] J.W. Bruce, The duals of generic hypersurfaces. Math. Scand. 49 (1981), no. 1, 36-60.

[5] J.W. Bruce, Wavefronts and parallels in Euclidean space. Math. Proc. Cambridge Philos. Soc. 93 (1983), no. 2, 323-333.

[6] J.W. Bruce, Generic geometry and duality. Singularities (Lille, 1991), 29-59, London Math. Soc. Lecture Note Ser., 201, Cambridge Univ. Press, Cambridge (1994).

[7] J.W. Bruce, Generic geometry, transversality and projections. J. London Math. Soc. (2) 49 (1994), no. 1, 183-194.

[8] L. Chen, Singularities of timelike Anti de Sitter Gauss images, Preprint.

[9] P.J. Giblin and G. Sapiro, Affine-invariant distances, envelopes and symmetry sets. Geom. Dedicata 71 (1998), no. 3, 237-261.

[10] S. Izumiya, D-H. Pei and T. Sano, The lightcone Gauss map and the lightcone developable of a spacelike curve in Minkowski 3-space, Glasgow. Math. J. 42 (2000), 75-89.

[11] S. Izumiya, D-H. Pei and T. Sano, Singularities of hyperbolic Gauss maps, Proc. London. Math. Soc. (3) 86 (2003), 485-512.

[12] S. Izumiya, M. Kikuchi and M. Takahashi, Global properties of spacelike curves in Minkowski 3-space, Journal of Knot theory and its Ramifications. 15 (2006), 869-881.

[13] S. Izumiya and M. Takahashi, Spacelike parallels and evolutes in Minkowski pseudo-spheres, J. Geometry and Physics. 57 (2007), 1569-1600. 
[14] S. Izumiya and T. Fusho, Lightlike surfaces of spacelike curves in de Sitter 3-space, Journal of Geometry. 88 (2008), 19-29.

[15] S. Izumiya, Timelike hypersurfaces in de Sitter space and Legendrian singularities, Journal of Mathematical Sciences. 144, No.1, (2007), 3789-3803.

[16] E.E. Landis, Tangential singularities. (Russian) Funktsional. Anal. i Prilozhen. 15 (1981), no. $2,36-49$.

[17] S. Lee, Timelike surfaces of constant mean curvature \pm 1 in anti-de Sitter 3 -space $\mathbb{H}_{1}^{3}(-1)$, Ann. Global Anal. Geom. 29 (2006), 361-407.

[18] J. N. Mather, Stability of $C^{\infty}$-mappings IV: classification of stable germs by $\mathbb{R}$ algebras, IHÉS. Publ. Math. 37 (1970), 223-248.

[19] J. Martinet, Singularities of Smooth Functions and Maps, London Math. Soc. Lecture Note Series. Cambridge Univ. Press. 58 (1982).

[20] D.K.H. Mochida, M.C. Romero Fuster and M.A.S. Ruas, The geometry of surfaces in 4-space from a contact viewpoint. Geom. Dedicata 54 (1995), no. 3, 323-332.

[21] D.K.H. Mochida, M.C. Romero-Fuster and M.A.S. Ruas, Osculating hyperplanes and asymptotic directions of codimension two submanifolds of Euclidean spaces. Geom. Dedicata 77 (1999), no. 3, 305-315.

[22] D.K.H. Mochida, M.C. Romero-Fuster and M.A.S. Ruas, Singularities and duality in the flat geometry of submanifolds of Euclidean spaces. Beitrage Algebra Geom. 42 (2001), no. 1, $137-148$.

[23] D.K.H. Mochida, M.C. Romero-Fuster and M.A.S. Ruas, Inflection points and nonsingular embeddings of surfaces in $\mathbf{R}^{5}$. Rocky Mountain J. Math. 33 (2003), no. 3, 995-1009.

[24] J. A. Montaldi, On contact between submanifolds, Michigan Math. J. 33 (1986), 81-85.

[25] J. A. Montaldi, Surfaces in 3-space and their contact with circles. J. Diff. Geom. 23 (1986), no. $2,109-126$.

[26] J. A. Montaldi, On generic composites of maps, Bull. London Math. Soc. 23 (1991), 81-85.

[27] B. O’Neil, Semi-Riemannian Geometry, Academic Press, New York, (1983).

[28] I.R. Porteous, The normal singularities of a submanifold. J. Diff. Geom. 5 (1971), 543-564.

[29] I.R. Porteous, Geometric differentiation. For the intelligence of curves and surfaces. Second edition. Cambridge University Press, Cambridge (2001).

[30] M.C. Romero Fuster, Sphere stratifications and the Gauss map. Proc. Roy. Soc. Edinburgh Sect. A 95 (1983), no. 1-2, 115-136.

[31] H. Whitney, On singularities of mappings of Euclidean spaces I, Ann. of Math. 62 (1955), $374-410$.

[32] V. M. Zakalyukin, Lagrangian and Legendrian singularities, Funct. Anal. Appl. 10 (1976), $23-31$.

[33] V. M. Zakalyukin, Reconstructions of fronts and caustics depending one parameter and versality of mappings, J. Sov. Math. 27 (1984), 2713-2735. 